\title{
SPITZER survey of dust grain processing in stable discs around binary post-AGB stars ${ }^{\star, \star \star}$
}

\author{
C. Gielen ${ }^{1}$, H. Van Winckel ${ }^{1}$, M. Min ${ }^{2}$, L. B. F. M. Waters ${ }^{1,2}$, and T. Lloyd Evans ${ }^{3}$ \\ 1 Instituut voor Sterrenkunde, Katholieke Universiteit Leuven, Celestijnenlaan 200D, 3001 Leuven, Belgium \\ e-mail: clio.gielen@ster. kuleuven . be \\ 2 Sterrenkundig Instituut "Anton Pannekoek", Universiteit Amsterdam, Kruislaan 403, 1098 Amsterdam, The Netherlands \\ 3 SUPA, School of Physics and Astronomy, University of St Andrews, North Haugh, St Andrews, Fife KY16 9SS, UK
}

Received 25 April 2008 / Accepted 3 September 2008

\begin{abstract}
Aims. We investigate the mineralogy and dust processing in the circumbinary discs of binary post-AGB stars using high-resolution TIMMI2 and SPITZER infrared spectra.

Methods. We perform a full spectral fitting to the infrared spectra using the most recent opacities of amorphous and crystalline dust species. This allows for the identification of the carriers of the different emission bands. Our fits also constrain the physical properties of different dust species and grain sizes responsible for the observed emission features.

Results. In all stars the dust is oxygen-rich: amorphous and crystalline silicate dust species prevail and no features of a carbon-rich component can be found, the exception being EP Lyr, where a mixed chemistry of both oxygen- and carbon-rich species is found. Our full spectral fitting indicates a high degree of dust grain processing. The mineralogy of our sample stars shows that the dust is constituted of irregularly shaped and relatively large grains, with typical grain sizes larger than $2 \mu \mathrm{m}$. The spectra of nearly all stars show a high degree of crystallinity, where magnesium-rich end members of olivine and pyroxene silicates dominate. Other dust features of e.g. silica or alumina are not present at detectable levels. Temperature estimates from our fitting routine show that a significant fraction of grains must be cool, significantly cooler than the glass temperature. This shows that radial mixing is very efficient is these discs and/or indicates different thermal conditions at grain formation. Our results show that strong grain processing is not limited to young stellar objects and that the physical processes occurring in the discs are very similar to those in protoplanetary discs.
\end{abstract}

Key words. stars: AGB and post-AGB - stars: evolution - stars: binaries: general - stars: circumstellar matter

\section{Introduction}

Post-AGB stars are low- and intermediate-mass stars that evolve rapidly from the Asymptotic Giant Branch (AGB) towards the Planetery Nebula (PNe) phase, before cooling down as white dwarves. During the previous AGB phase, the star has undergone severe mass loss, leaving behind a slowly expanding circumstellar dust shell. Depending on the dilution in the line-ofsight, the expected spectral energy distribution (SED) is doublepeaked, with the first UV-visible peak indicative of the central star and the second infrared peak coming from thermal emission of the cool dust in the circumstellar environment (CE). The release of the IRAS All-Sky Survey mission made a largescale identification of post-AGB stars on the basis of their infrared colours possible (Lloyd Evans 1985; Hrivnak et al. 1989;

\footnotetext{
* Based on observations obtained at the European Southern Observatory (ESO), La Silla, observing program 072.D-0263, on observations made with the $1.2 \mathrm{~m}$ Flemish Mercator telescope at Roque de los Muchachos, Spain, the $1.2 \mathrm{~m}$ Swiss Euler telescope at La Silla Chile and on observations made with the SPITZER Space Telescope (program id 3274), which is operated by the Jet Propulsion Laboratory, California Institute of Technology under a contract with NASA.

$\star \star$ Appendices A and B are only available in electronic form at http://www . aanda.org
}

Oudmaijer et al. 1992). More recent surveys on post-AGB stars include Szczerba et al. (2007), who present the latest catalogue of Galactic post-AGB stars with almost 400 objects.

IRAS colour-colour diagrams revealed a large sample of stars that did not show the expected double-peaked SED (Lloyd Evans 1985; De Ruyter et al. 2006). Instead, they show a strong near-IR excess, pointing to the presence of hot dust in the system, while the central stars are currently too hot to have an ongoing dusty mass loss. Correlations with optical databases of variable stars point to the presence of pulsating stars that also show this specific IR-excess. These RV Tauri stars are luminous evolved stars that cross the Population II Cepheid instability strip and populate a well-defined part of the IRAS colour-colour diagram (Lloyd Evans 1985; Raveendran 1989; Lloyd Evans 1999). Recent interferometric studies (Deroo et al. 2007a,b) prove that the circumstellar emission originates from a very compact region and that the SEDs of these stars are well modelled with a passive 2D disc model (Dullemond \& Dominik 2004; Deroo et al. 2007a; Gielen et al. 2007). The spectral slope of the submillimetre SED points to the presence of large, up to $\mu \mathrm{m}$ and $\mathrm{cm}$ sized, grains in these discs (De Ruyter et al. 2006; Gielen et al. 2007), which have settled to form a cool disc midplane. The inner radius of these discs is determined by the dust sublimation radius. The hot inner rim is puffed up by gas presssure from the central 
star and radiates mainly in the near-IR, while the outer parts of the disc can be strongly flared and are responsible for the strong absorption and re-radiation of the stellar light. In a few cases the direct confirmation of the Keplerian kinematics of the circumstellar discs comes from resolved interferometric CO measurements (Bujarrabal et al. 1988, 2005, 2007). Recent studies have shown that these post-AGB stars are very likely all binaries (Maas et al. 2002, 2003; Van Winckel et al. 1999; Van Winckel 2007; Gielen et al. 2007).

Famous examples of these objects include HD 44179, the central star of the Red Rectangle. It is a carbon-rich post-AGB object for which the binarity was proven by Van Winckel et al. (1995). The star is surrounded by an oxygen-rich disc (Waters et al. 1998) and is resolved in ground-based high spatial resolution imaging (Roddier et al. 1995; Men'shchikov et al. 2002) as well as in HST optical images (Cohen et al. 2004). The disc is also resolved in interferometric $\mathrm{CO}$ measurements, where the Keplerian velocity of the disc was detected (Bujarrabal et al. 2005).

Infrared spectroscopy is the ideal tool to study the physicochemical characteristics of the circumstellar material, since it samples resonances of the most dominant dust species and important ro-vibrational bands of dominant molecules. Spectroscopic data obtained with the Infrared Space Observatory (ISO) allowed for the first time to study the mineralogy of circumstellar environments of objects in different evolutionary stages (e.g. Waters et al. 1996; Waelkens et al. 1996). More advanced studies of the CE of young stellar objects (e.g. van Boekel et al. 2005; Lisse et al. 2007) and mass-losing objects (Molster et al. 2002a,b,c) further identified the dominant dust species and grain sizes of the circumstellar dust.

Infrared spectral studies of post-AGB objects (Van Winckel 2003, and references therein) allowed for the detection of different $\mathrm{CE}$ chemistries. The more typical C-rich post-AGB stars are characterised by a strong amorphous $\mathrm{SiC}$ feature at $11.3 \mu \mathrm{m}$. In these stars, a strong unidentified $21 \mu \mathrm{m}$ feature can sometimes be found (Kwok et al. 1989). In addition to the detection of O-rich post-AGB stars, dominated by silicate species, surprisingly also mixed chemistries were found, showing features of both O-rich and C-rich dust species.

Young stellar objects are the natural environment to study circumstellar disc physics, but previous studies of the mineralogy of discs around the few brightest infrared evolved objects with ISO (Molster et al. 1999, 2002a,b,c) show strong dust processing, with oxygen-rich and highly crystalline dust. Molster et al. $(2002 a, b, c)$ found that a high degree of crystallisation is indicative for the presence of a stable disc, and not a dusty outflow.

In our pilot study (Gielen et al. 2007), based on SPITZER-IRS and ISO data, we investigate the mineralogy and spectral energy distribution of two post-AGB stars, RU Cen and ACHer. The spectra of these stars are extremely similar and show a strong resemblance to the infrared spectrum of the solarsystem comet Hale-Bopp and young stellar objects, such as HD 100546. The observed crystalline emission features are well modelled using magnesium-rich crystalline silicates. The grains are irregularly shaped, with grain sizes of $0.1 \mu \mathrm{m}$ and $1.5 \mu \mathrm{m}$. Both hot and cool grains are necessary to reproduce the observed spectrum. The spectral energy distributions of both stars were fitted using a 2D passive disc model (Dullemond \& Dominik 2004). The discs surrounding these objects start at 35 AU from the central star, which is well beyond the dust sublimation radius. The outer radius is less well constrained, but extends till about $300 \mathrm{AU}$. The discs are strongly flared.
In this work we study the mineralogy of the discs in a large sample of post-AGB binaries and perform a detailed fitting of the observed emission features. We have observed 21 binary postAGB objects with the SPITZER-IRS spectrograph and look for relations between the dust parameters and stellar characteristics.

The outline of this paper is as follows: in Sects. 2 and 3 we introduce our programme stars and the different observation and reduction strategies used. Section 4 contains the construction of the spectral energy distributions and the total extinction determination. In Sect. 5 we give a general overview of the spectra and the observed emission features. The profile and full spectral fitting is presented in Sects. 6 and 7. The discussion and conclusion of our analysis are given in Sects. 8 and 9.

\section{Programme stars}

From the total sample of 51 published binary post-AGB stars likely surrounded by a stable disc (De Ruyter et al. 2006), we selected the 21 stars with fluxes below the saturation limit of the SPITZER-IRS spectrograph. Spectral types range from A to M.

Our radial velocity monitoring program, using the CORALIE spectrograph attached to the $1.2 \mathrm{~m}$ Swiss Euler Telescope at the ESO La Silla Observatory, is still ongoing but we have already found orbital parameters for 15 of our 21 program stars. Orbital periods range from 100 up to more than 2000 days (Van Winckel 2007). The objects for which we do not yet have orbital parameters, have pulsational amplitudes which are too large for a straightforward detection of the binary motion. The results of the radial velocity monitoring program will be subject of a forthcoming paper.

The orbital periods indicate that strong binary interaction must have occurred during the late stages on the AGB. The binaries are now not in contact but the orbits are too small to accommodate a full-grown AGB star. The discs are all circumbinary since all orbits lie well within the dust sublimation radius. The distribution of the mass-functions gives a range in minimal mass for the companion between $1 M_{\odot}$ and $2 M_{\odot}$. The companion stars are likely to be unevolved main sequence stars (e.g. Gielen et al. 2007; Van Winckel 2007).

These binary post-AGB stars are characterised by a depletion pattern in their photospheres (Giridhar et al. 1994, 1998, 2000; Gonzalez et al. 1997a,b; Van Winckel et al. 1998; Maas et al. 2005). This abundance pattern is the result of gas-dust separation followed by reaccretion of the gas, which is poor in refractory elements. Waters et al. (1992) proposed that the most likely circumstance for this process to occur is when the dust is trapped in a circumstellar disc. Photospheric chemical studies of post-AGB candidates in the LMC have revealed that there also, depletion patterns are common (Reyniers \& Van Winckel 2007). The observations in the recent release of the SAGE database (Meixner et al. 2006) showed that these depleted LMC sources have infrared excesses similar to the Galactic binary post-AGB stars, and are therefore thought to be disc sources as well.

The disc formation itself is badly understood. Possible formation scenarios of the discs include a wind capture scenario (e.g. Mastrodemos \& Morris 1999), or a formation scenario through non-conservative mass transfer in an interacting binary. In the first scenario, the AGB wind is captured by the companion. In the second scenario, which is still not very well explored theoretically, the disc formation precedes the dust-grain formation and it is likely that the thermal history of the grains was very different from that in normal AGB winds. This could lead to very different chemico-physical properties during formation. The sizes of the orbits suggest this scenario to be more likely. 
Table 1. The name, equatorial coordinates $\alpha$ and $\delta(\mathrm{J} 2000)$, spectral type, effective temperature $T_{\text {eff }}$, surface gravity $\log g$ and metallicity [Fe/H] of our sample stars. For the model parameters we refer to De Ruyter et al. (2006). Also given is the orbital period (see references in De Ruyter et al. 2006; Gielen et al. 2007). The total reddening $E(B-V)_{\mathrm{tot}}$, the energy ratio $L_{\mathrm{IR}} / L_{*}$ and the calculated distance, assuming a luminosity of $L_{*}=5000 \pm 2000 L_{\odot}$. Distances marked with * are upper limits due to the aspect angle of the disc.

\begin{tabular}{llrrrrrrrrr}
\hline \hline$N^{\circ}$ & Name & $\begin{array}{r}\alpha(\mathrm{J} 2000) \\
(\mathrm{h} \mathrm{m} \mathrm{s})\end{array}$ & $\begin{array}{r}\delta(\mathrm{J} 2000) \\
\left({ }^{\circ}{ }^{\prime \prime}\right)\end{array}$ & $\begin{array}{r}T_{\text {eff }}(\mathrm{K}) \\
(\mathrm{cgs})\end{array}$ & $\begin{aligned} \log g \\
(\mathrm{Fe} / \mathrm{H}]\end{aligned}$ & $\begin{array}{r}P_{\text {orbit }} \\
\text { (days })\end{array}$ & $E(B-V)_{\text {tot }}$ & $\begin{array}{r}L_{\mathrm{IR}} / L_{*} \\
(\%)\end{array}$ & $\begin{array}{r}d \\
(\mathrm{kpc})\end{array}$ \\
\hline 1 & EP Lyr & 191817.5 & +275038 & 7000 & 2.0 & -1.5 & & $0.52 \pm 0.01$ & $3 \pm 0$ & $4.1 \pm 0.8$ \\
2 & HD 131356 & 145700.7 & -685023 & 6000 & 1.0 & -0.5 & 1490 & $0.20 \pm 0.01$ & $50 \pm 2$ & $3.0 \pm 0.6$ \\
3 & HD 213985 & 223527.5 & -171527 & 8250 & 1.5 & -1.0 & 259 & $0.27 \pm 0.01$ & $24 \pm 1$ & $3.1 \pm 0.6$ \\
4 & HD 52961 & 070339.6 & +104613 & 6000 & 0.5 & -4.8 & 1310 & $0.06 \pm 0.01$ & $12 \pm 1$ & $2.1 \pm 0.4$ \\
5 & IRAS 05208-2035 & 052259.4 & -203253 & 4000 & 0.5 & 0.0 & 236 & $0.00 \pm 0.00$ & $38 \pm 2$ & $3.9 \pm 0.8$ \\
6 & IRAS 09060-2807 & 090810.1 & -281910 & 6500 & 1.5 & -0.5 & 371 & $0.57 \pm 0.02$ & $63 \pm 3$ & $5.4 \pm 1.1$ \\
7 & IRAS 09144-4933 & 091609.1 & -494606 & 5750 & 0.5 & -0.5 & 1770 & $1.99 \pm 0.05$ & $53 \pm 5$ & $2.7 \pm 0.6$ \\
8 & IRAS 10174-5704 & 101918.1 & -571936 & & & & 323 & & & \\
9 & IRAS 16230-3410 & 162620.3 & -341712 & 6250 & 1.0 & -0.5 & & $0.56 \pm 0.02$ & $60 \pm 3$ & $6.1 \pm 1.2$ \\
10 & IRAS 17038-4815 & 170736.3 & -481908 & 4750 & 0.5 & -1.5 & 1381 & $0.22 \pm 0.02$ & $69 \pm 5$ & $4.5 \pm 1.0$ \\
11 & IRAS 17243-4348 & 172756.1 & -435048 & 6250 & 0.5 & 0.0 & 484 & $0.59 \pm 0.02$ & $68 \pm 4$ & $3.8 \pm 0.8$ \\
12 & IRAS 19125+0343 & 191500.8 & +034841 & 7750 & 1.0 & -0.5 & 517 & $1.08 \pm 0.02$ & $52 \pm 3$ & $1.8 \pm 0.4$ \\
13 & IRAS 19157-0247 & 191822.5 & -024209 & 7750 & 1.0 & 0.0 & 120.5 & $0.68 \pm 0.01$ & $63 \pm 2$ & $4.2 \pm 0.9$ \\
14 & IRAS 20056+1834 & 200754.8 & +184257 & 5850 & 0.7 & -0.4 & & $0.51 \pm 0.02$ & $905 \pm 42$ & $10.9 \pm 2.3$ \\
15 & RU Cen & 120923.7 & -452535 & 6000 & 1.5 & -2.0 & 1489 & $0.55 \pm 0.01$ & $13 \pm 1$ & $2.3 \pm 0.5$ \\
16 & SAO 173329 & 071608.3 & -232702 & 7000 & 1.5 & -0.8 & 115.9 & $0.39 \pm 0.01$ & $36 \pm 1$ & $6.5 \pm 1.3$ \\
17 & ST Pup & 064856.4 & -371633 & 5750 & 0.5 & -1.5 & 410 & $0.00 \pm 0.00$ & $55 \pm 1$ & $5.7 \pm 1.2$ \\
18 & SU Gem & 061400.8 & +274212 & 5750 & 1.125 & -0.7 & & $0.58 \pm 0.02$ & $111 \pm 7$ & $4.8 \pm 1.0^{*}$ \\
19 & SX Cen & 122112.6 & -491241 & 6000 & 1.0 & -1.0 & 600 & $0.32 \pm 0.02$ & $34 \pm 2$ & $3.8 \pm 0.7$ \\
20 & TW Cam & 042048.1 & +572626 & 4800 & 0.0 & -0.5 & & $0.40 \pm 0.02$ & $42 \pm 3$ & $3.2 \pm 0.6$ \\
21 & UY CMa & 061816.4 & -170235 & 5500 & 1.0 & 0.0 & & $0.00 \pm 0.00$ & $89 \pm 3$ & $9.6 \pm 2.0^{*}$ \\
\hline
\end{tabular}

We may witness the formation of a circumbinary disc by Roche-Lobe overflow in the evolved binary system SS Lep. The optical/IR interferometric observables can be best understood, assuming a Roche-lobe filling $M$ star in a system with a circumsystem disc (Verhoelst et al. 2007).

Our program stars are therefore all likely binaries which are surrounded by a dusty stable disc. This disc seems to have an important impact on the objects and in this paper we focus on the analysis of the IR spectra as probes for the disc physics.

\section{Observations and data reduction}

\subsection{SPITZER}

High- and low-resolution spectra for 21 post-AGB stars were obtained using the Infrared Spectrograph (IRS; Houck et al. 2004) aboard the SPITZER Space Telescope (Werner et al. 2004) in February 2005. The spectra were observed using combinations of the short-low (SL), short-high (SH) and long-high (LH) modules. SL $(\lambda=5.3-14.5 \mu \mathrm{m})$ spectra have a resolving power of $R=\lambda / \Delta \lambda \sim 100$, SH $(\lambda=10.0-19.5 \mu \mathrm{m})$ and LH $(\lambda=19.3-37.0 \mu \mathrm{m})$ spectra have a resolving power of $\sim 600$. Exposure times were chosen to achieve a S/N ratio of around 400 for the high-resolution modes, which we complemented with short exposures in low-resolution mode with a $\mathrm{S} / \mathrm{N}$ ratio around 100 , using the first generation of the exposure time calculator of the call for proposals.

The spectra were extracted from the SSC raw data pipeline version S13.2.0 products, using the c2d Interactive Analysis reduction software package (Kessler-Silacci et al. 2006; Lahuis et al. 2006). This data processing includes bad-pixel correction, extraction, defringing and order matching. Individual orders are corrected for offsets, if necessary, by small scaling corrections to match the bluer order.

\section{2. $T I M M I 2$}

For stars where we lack the SPITZER IRS-SH observations we obtained additional ground-based N-band infrared spectra with the Thermal Infrared Multi Mode Instrument 2 (TIMMI2, Reimann et al. 2000; Käufl et al. 2003), mounted on the $3.6 \mathrm{~m}$ telescope at the ESO La Silla Observatory. The low-resolution $(R \sim 160) \mathrm{N}$ band grism was used in combination with a 1.2 arcsec slit, the pixel scale in the spectroscopic mode of TIMMI 2 is 0.45 arcsec. For the reduction of the spectra we used the method described in van Boekel et al. (2005). We scaled the TIMMI2 spectra to the SPITZER spectra and found a very good agreement in spectral shape between the two data sets.

\section{Spectral energy distribution}

For all sample stars spectral energy distributions were updated from the photometric data as given by De Ruyter et al. (2006). The resulting SEDs are presented in Figs. B.1 and B.2. The total extinction $E(B-V)_{\text {tot }}$ was determined by dereddening the observed photometry, using the average extinction law of Savage $\&$ Mathis (1979). The relation between $E(B-V)_{\text {tot }}$ and $A_{V}$ is given by $A_{V}=R_{V} \times E(B-V)$, with a typical value for $R_{V}=3.1$ (Savage \& Mathis 1979). Minimising the difference between the dereddened observed optical fluxes and the appropriate Kurucz model (Kurucz 1979) gives the total colour excess $E(B-V)_{\text {tot }}$ (Table 1). Model parameters for our sample stars are given in Table 1 and are based on the analysis of high-resolution spectra as given by the literature (see De Ruyter et al. 2006, for references). A considerable fraction of our sample stars show a photometric variability due to pulsation, so we use only photometric maxima for the SED construction.

The errors on the value for $E(B-V)_{\text {tot }}$ are calculated using a Monte-Carlo simulation on the photometric data. We use an error of 0.05 for the photometric measurements in a Gaussian distribution. Since we do not know the distances to the sources, 


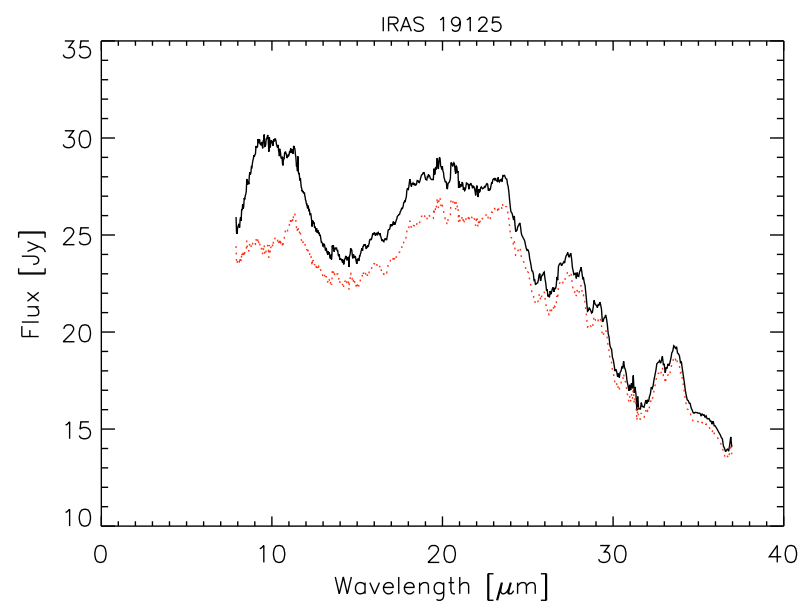

Fig. 1. Comparison between the dereddened (black solid line) and reddened (red dotted line) of IRAS 19125. Dereddening mainly influences the spectral signature in the $10 \mu \mathrm{m}$ region.

we adopt a likely luminosity for evolved low-gravity objects of $L_{*}=5000 \pm 2000 L_{\odot}$. The distance to IRAS 20056 is likely overestimated since visible light from this source probably reaches us only by scattering through a nearly edge-on optically thick disc (Menzies \& Whitelock 1988; Gledhill et al. 2001).

We also corrected the infrared spectra for reddening by extending the average extinction law of Savage \& Mathis (1979) with the theoretical extinction law of Steenman \& Thé (1989, 1991) and by using the derived total extinction values. This is done under the assumption that the extinction is fully due to interstellar extinction. Since the total extinction probably consists of both an interstellar and a circumstellar component, the applied dereddening is thus a maximal correction.

Dereddening the infrared spectra mainly influences the shape of the silicate feature around $9.8 \mu \mathrm{m}$ (see Fig. 1). Since our total extinction values are on average quite small, the effect of deredding the infrared spectra is negligible for most stars. For stars with a higher value for $E(B-V)_{\text {tot }}$, like IRAS 09144 and IRAS 19125, one has to be careful interpreting the shape of the silicate feature, since the partitioning between interstellar and circumstellar reddening is not known at this point.

We compute the energy ratio $L_{\mathrm{IR}} / L_{*}$ to determine the amount of energy reprocessed by the CE (Table 1). $80 \%$ of our stars have a ratio $L_{\mathrm{IR}} / L_{*}$ of $30 \%$ and higher, showing that the absorption and re-radiation of stellar light by the CE is extremely efficient.

\section{General overview}

Figures B.8 till B.10 show the wide range in observed spectra. All spectra are characterised by many distinct spectral structures in emission over a smooth continuum. There is quite some variety over the sample, with different continuum slopes, but for nearly all stars the spectrum is dominated by emission bands that can be attributed to silicate dust species.

In a few stars only, gas phase emission is detected in the form of bandhead emission of ${ }^{12} \mathrm{CO}_{2}$ and ${ }^{13} \mathrm{CO}_{2}$. These stars are HD 52961, EP Lyr and IRAS 10174.

Unique to our sample is the spectrum of EPLyr in the $7-20 \mu \mathrm{m}$ region. This spectral range is dominated by a strong and narrow $11.3 \mu \mathrm{m}$ emission peak and a complex plateau with narrow emission features in the $14-18 \mu \mathrm{m}$ region. The strongly asymmetric band between $7 \mu \mathrm{m}$ and $10 \mu \mathrm{m}$ is real as well. These features are observed in C-rich evolved stars and in the

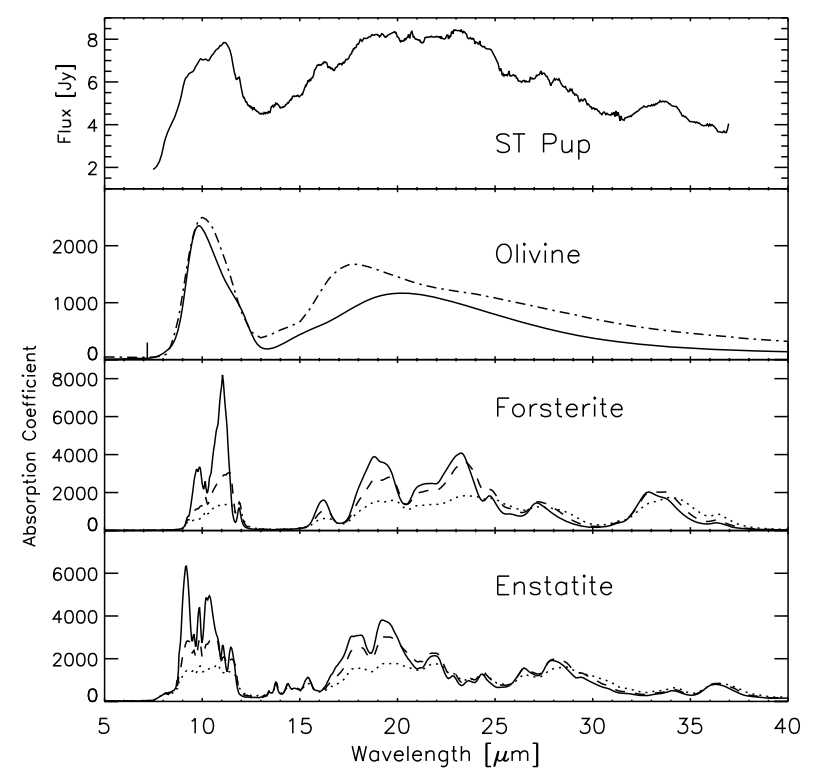

Fig. 2. The mass absorption coefficients $\left[\mathrm{cm}^{2} / \mathrm{g}\right]$ of forsterite and enstatite in GRF approximation. Grain sizes of $0.1 \mu \mathrm{m}$ (full line), $2.0 \mu \mathrm{m}$ (dashed line) and $4.0 \mu \mathrm{m}$ (dotted line) are plotted. The third box shows olivine in $0.1 \mu \mathrm{m}$ GRF approximation. The $\mathrm{Mg}$-rich member $\left(\mathrm{Mg}_{2} \mathrm{SiO}_{4}\right)$ is plotted in full line, the standard $\mathrm{Mg}$-Fe member $\left(\mathrm{MgFeSiO}_{4}\right)$ is plotted in dot-dashed line. As an example we also plotted the SPITZER spectrum of one of our sample stars, ST Pup.

carbon-rich component of the ISM and are associated with $\mathrm{PAH}$ emission and the associated $\mathrm{CH}$-out-of-plane and C-CC bending modes (e.g. Van Kerckhoven et al. 2000; Hony et al. 2001; Peeters et al. 2002). Longward of $18 \mu \mathrm{m}$, the spectrum is dominated by silicate emission. EP Lyr, is the only object in our sample displaying a mixed chemistry. It is remarkable that in our considerable sample of post-AGB stars, only one possible post-carbon star is found!

\subsection{Silicate dust features: identification}

Amorphous and crystalline silicates are the most commonly found dust species in the interstellar and circumstellar environment (Molster et al. 2002a,b,c; Kemper et al. 2004; Min et al. 2007). To describe the glassy and crystalline silicates with an olivine and pyroxene stoichiometry we will use the commonly used term "amorphous and crystalline olivine and pyroxene". Amorphous olivine $\left(\mathrm{Mg}_{2 x} \mathrm{Fe}_{2(1-x)} \mathrm{SiO}_{4}\right.$, where $0 \leq x \leq 1$ denotes the magnesium content) has very prominent broad features around $9.8 \mu \mathrm{m}$ and $18 \mu \mathrm{m}$ (see Fig. 2), which are easily detected in our spectra. These features (also called the $10 \mu \mathrm{m}$ and $20 \mu \mathrm{m}$ features) arise respectively from the $\mathrm{Si}-\mathrm{O}$ stretching mode and the O-Si-O bending mode. For large grains the $9.8 \mu \mathrm{m}$ feature gets broader and shifts to redder wavelengths. Amorphous pyroxene $\left(\mathrm{Mg}_{x} \mathrm{Fe}_{1-x} \mathrm{SiO}_{3}\right)$ shows a $10 \mu \mathrm{m}$ feature similar to that of amorphous olivine, but shifted towards shorter wavelengths. Also the shape of the $20 \mu \mathrm{m}$ feature is slightly different.

The $\mathrm{Mg}$-rich end members of crystalline olivine and pyroxene, forsterite $\left(\mathrm{Mg}_{2} \mathrm{SiO}_{4}\right)$ and enstatite $\left(\mathrm{MgSiO}_{3}\right)$, show strong but narrow features at distinct wavelengths around 11.3-16.2-19.7-23.7-28-33.6 $\mu \mathrm{m}$ (see Fig. 2), making them easily identifiable in our spectra. Forsterite condenses directly from the gas-phase at high temperatures $(\approx 1500 \mathrm{~K})$ or it may form by thermal annealing of amorphous silicates, diffusing the iron out of the lattice-structure. Enstatite can form in the 
gas-phase from a reaction between forsterite and silica $\left(\mathrm{SiO}_{2}\right)$, or it may also form by a similar thermal annealing process as forsterite (Bradley et al. 1983; Tielens et al. 1997). Laboratory experiments have indicated that silica can be formed when amorphous silicates anneal to forsterite (e.g. Fabian et al. 2000). No evidence for the presence of silica, with strong features around 9 and $21 \mu \mathrm{m}$, can be found in our spectra. There is also no evidence for the presence of Fe-rich crystalline silicates, like fayalite $\left(\mathrm{Fe}_{2} \mathrm{SiO}_{4}\right)$.

Nearly all stars show strong crystalline dust features, both at short and long wavelengths, showing that both hot and cool crystallines seem to be abundant.

In our spectra, the amorphous silicate dust seems to peak at $20 \mu \mathrm{m}$, rather than $18 \mu \mathrm{m}$, as would be expected from synthetic spectra of amorphous silicates. This could point to the presence of Mg-rich amorphous dust, which also shows this shift to redder wavelengths (see Fig. 2).

\subsection{Mean spectra and complexes}

Different complexes at 10,14, 16, 19, 23 and $33 \mu$ m can be identified in our spectra. In order to study the systematics between these complexes in our spectra we compare them to a mean spectrum in that region. The mean complex spectra were obtained by adding continuum subtracted spectra of sample sources with clear spectral structures, using a weighing factor proportional to the $\mathrm{S} / \mathrm{N}$ in that spectral region. The continuum was determined by linearly interpolating between the beginning and end of the studied region. The $10 \mu \mathrm{m}$ complex is discussed below, other complexes are discussed in the appendix (Sect. B.1). The mean complex spectra are shown in Figs. B.3 to B.7. We also plot the mass absorption coefficients of forsterite and enstatite in every complex.

\subsubsection{The $10 \mu \mathrm{m}$ complex $(8-13 \mu \mathrm{m})$}

In Fig. 3 we plot the continuum divided spectra in the $10 \mu \mathrm{m}$ region, ordered by peak value. We plot $1+F_{v, \mathrm{cs}} /\left\langle F_{v, \mathrm{c}}\right\rangle$, where $F_{v, \mathrm{cs}}$ is the continuum subtracted spectrum $\left(F_{v}-F_{v, \mathrm{c}}\right)$ and $\left\langle F_{v, \mathrm{c}}\right\rangle$ is the mean of the continuum. The continuum was determined by linearly interpolating between 7.5 and $13 \mu \mathrm{m}$. This method preserves the shape of the emission band and allows a good comparison between the profiles themselves. The figure clearly illustrates the very wide variety of the spectral appearance of the warm silicates, and because of this large variety a mean spectrum was not constructed. We do not find a relation between the strength and the shape of the silicate feature.

In Fig. 4 we show the continuum subtracted flux at 11.3 and $9.8 \mu \mathrm{m}$ versus the peak to continuum ratio of the $10 \mu \mathrm{m}$ silicate feature. The $11.3 / 9.8 \mu \mathrm{m}$ ratio is a measure for the amount of processing that the dust has undergone, where the peak/continuum ratio is a measure for the typical grain size. We find a high degree of crystallisation $(11.3 / 9.8 \mu \mathrm{m}$ ratio) for all sources, but rather small peak to continuum values in general. EPLyr is a clear outlier with a very strong $11.3 / 9.8 \mu \mathrm{m}$ ratio, due to a very sharp peak at $11.3 \mu \mathrm{m}$ which is due to PAH emission. No clear correlation, and thus no evolutionary trend, is observed between the shape and the strength of the $10 \mu \mathrm{m}$ feature in our sample stars, however. This is in contrast with the clear correlation that is seen in young stellar objects (van Boekel et al. 2003, 2005), where stars with a strong feature have low $11.3 / 9.8 \mu \mathrm{m}$ ratios and stars with a weaker silicate feature show higher $11.3 / 9.8 \mu \mathrm{m}$ ratios. Sources that display a strong

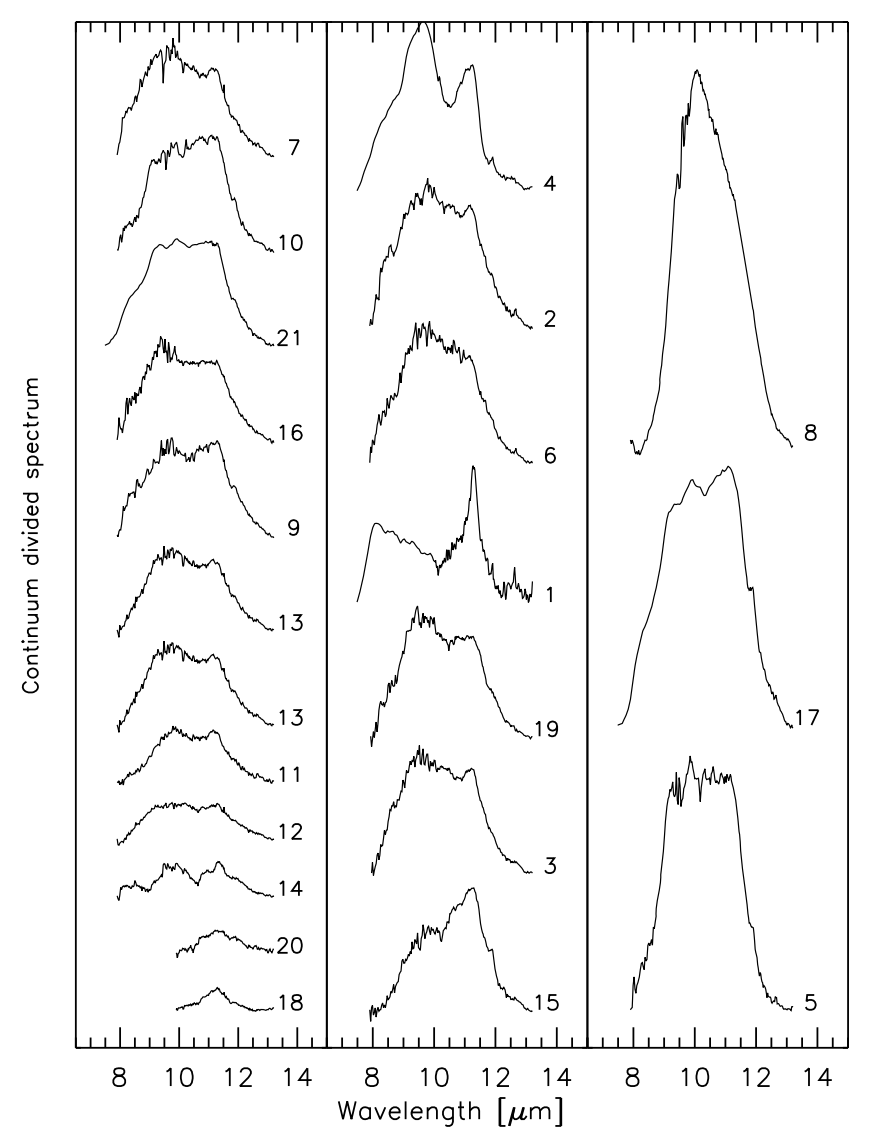

Fig. 3. Continuum divided spectra in the $10 \mu \mathrm{m}$ region, ordered by peak value. The peak value increases from left to right and from bottom to top. The numbering corresponds with the star numbers as given in Table 1. For SU Gem and TW Cam we lack flux points in the $7.5 \mu \mathrm{m}$ region to perform a good continuum determination.

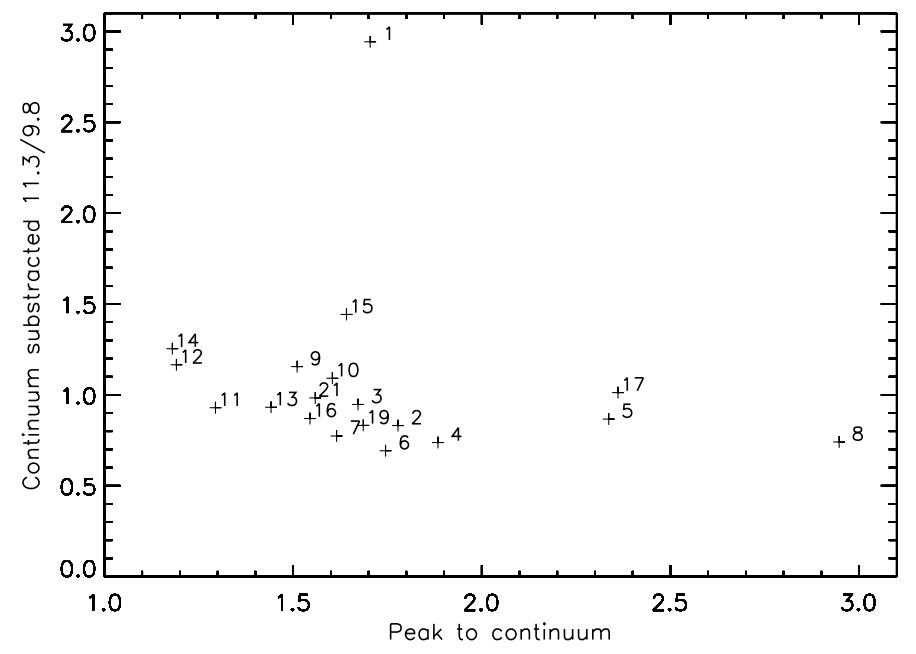

Fig. 4. Continuum subtracted flux at 11.3 and $9.8 \mu \mathrm{m}$ versus the peak to continuum ratio of the $10 \mu \mathrm{m}$ silicate feature. SUGem and TW Cam are not plotted since for these stars we lack a good continuum determination.

emission feature show rather unprocessed silicate band, similar to the ISM profile, with little evidence for crystalline grains. Sources with a weak $10 \mu \mathrm{m}$ profile show a broader and flatter silicate feature, dominated by larger grains. 


\section{Analysis: profile fitting}

To fit the observed emission features with synthetic spectra, mass absorption coefficients for different dust species need to be calculated. The conversion from laboratory measured optical constants of dust to mass absorption coefficients is not straightforward and is largely dependent on the adopted size, shape, structure and chemical composition of the dust (Min et al. 2003, 2005a). These different dust approximations result in very different predicted emission feature profiles, with a clear division between the synthetic spectra of homogeneous and irregular grains. If one would assume homogeneous spherical particles, one could use Mie theory (Aden \& Kerker 1951; Toon \& Ackerman 1981). However, cosmic dust grains are not perfect spheres, so we have to reside to other methods. Examples of irregular dust approximations are CDE (continuous distribution of ellipsoids, Bohren et al. 1983), GRF (Gaussian random field particles, e.g. Grynko \& Shkuratov 2003; Shkuratov \& Grynko 2005) and DHS (distribution of hollow spheres, Min et al. 2003, 2005a) grain shapes. The DHS shaped particles are further characterised by the fraction of the total volume occupied by the central vacuum inclusion, $f$, over the range $0<f<f_{\max }$. The value of $f_{\max }$ reflects the degree of irregularity of the particles (Min et al. 2003, 2005a). Cross sections in CDE are computed under the assumption that the grains are in the Rayleigh limit (that the grains are much smaller than the wavelength of radiation, thus smaller than $0.1 \mu \mathrm{m}$ ), and do not allow the study of grain growth. The spectra for the GRF particles were computed using the Discrete Dipole Approximation (Draine 1988). More details on the particle shapes and the computations of the spectra can be found in Min et al. (2007) and Min 2008 (in preparation). Refractory indices for the materials used are taken from Servoin \& Pirou (1973), Dorschner et al. (1995), Henning \& Stognienko (1996), Jaeger et al. (1998).

Not only grain shape, but also grain size, has a profound influence on the mass absorption coefficients. Since a circumstellar disc is the perfect environment for grain growth to occur, we also study the effect of different grain sizes on the observed emission profiles. Different grain sizes produce emission features at different central wavelengths and with different profiles, as shown in Fig. 2. With increasing grain size, emission features will become weaker and eventually disappear, leaving mainly a contribution to the thermal continuum. This effect is already considerable for grain radii $a>2 \mu \mathrm{m}$. Previous studies (Bouwman et al. 2001; Honda et al. 2004) in the $10 \mu \mathrm{m}$ region have shown that the variety of emission features can be described using two typical grain sizes: generally $0.1 \mu \mathrm{m}$ to describe the grains with radii $<1 \mu \mathrm{m}$, and $1.5-2 \mu \mathrm{m}$ for larger grains.

Comparing the observed crystalline emission features with calculated synthetic spectra (Fig. 5), we find that irregular grains are needed to explain the dust profiles. When we look at the prominent forsterite features at 16-19-23.7-33.6 $\mu \mathrm{m}$, we find a good fit is achieved using grains in CDE, GRF or DHS (with $f=1.0$ ). None of the tested dust shapes proves an accurate fit to the, in some stars very strong, forsterite $16 \mu \mathrm{m}$ feature, which seems to be shifted to bluer wavelengths in the observed spectra. This was also seen in the disc spectra of Molster et al. (2002a). The best fit to this feature is achieved using dust in GRF approximation. The fit of the $23.7 \mu \mathrm{m}$ feature is strongly improved when using large grains in DHS. The GRF approximation does not result in a good fit to the $33.6 \mu \mathrm{m}$ feature, which is the purest forsterite feature, since at other wavelengths the features may be blended with e.g. enstatite emission features. Both CDE and DHS reproduce the shape of this feature, but since the CDE
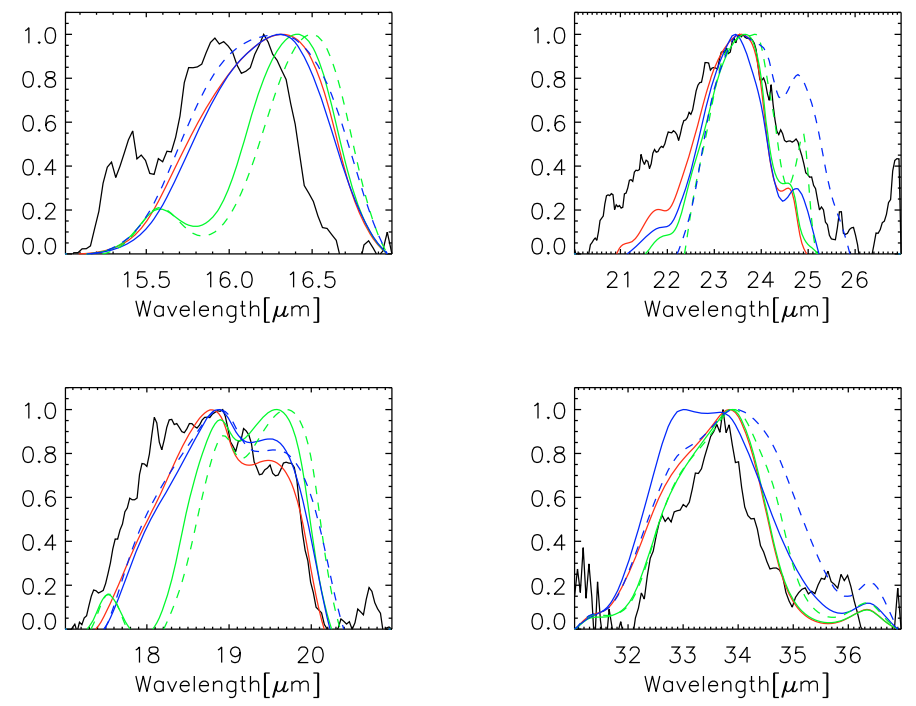

Fig. 5. Normalised and continuum subtracted emission features of the mean of our sample stars, together with mass absorption coefficients for different forsterite shape distributions. CDE grains are plotted in red, GRF in blue and DHS in green. $0.1 \mu \mathrm{m}$ grains are plotted in full lines, $2 \mu \mathrm{m}$ grains in dashed.

approximation does not allow us to study the effect of large grains we use GRF and DHS approximations in our fitting routine.

A similar approach to determine the best enstatite or amorphous silicate dust approximations is not straightforward. Enstatite emission features are mostly blended with forsterite features, but in the $14 \mu \mathrm{m}$ region there are some small unblended features. These are ideal features to trace the enstatite fraction of the grains. The central wavelengths of these peaks are at 13.8, 14.4 and $15.4 \mu \mathrm{m}$, with the $15.4 \mu \mathrm{m}$ feature being the most prominent. The sample sources that show clear emission in this region all show the $13.8 \mu \mathrm{m}$ feature and in lesser degree the $15.4 \mu \mathrm{m}$ feature. The $14.4 \mu \mathrm{m}$ feature is absent but a rather strong unidentified feature appears around $14.8 \mu \mathrm{m}$. In order to keep the fitting homogeneous we will also use GRF and DHS dust approximations to describe the amorphous and crystalline pyroxene dust species.

\section{Full spectral fitting}

\subsection{Method}

For an exact spectral modelling, full $2 \mathrm{D}$ radiative transfer in a realistic disc model should be studied. Such models are not yet available so as a first approximation we assume the emission features to be formed in the thin surface layer of the disc. Assuming the flux originates from an optically thin region, which is motivated by the fact we see the dust features in emission, we can make linear combinations of the absorption profiles to calculate the model spectrum.

This approach is a first approximation but allows for a general study of trends in dust shapes, grain sizes and processing in these discs. Disc models like the one of Dullemond \& Dominik (2004), which we used in our pilot study to fit the SED of RU Cen and AC Her, do not allow for a detailed spectral modelling. The code computes the temperature structure and density of the disc. The vertical scale height of the disc is computed by an iteration process, demanding vertical hydrostatic equilibrium. Other important processes, like dust settling and turbulent 
mixing, probably also occur in these discs but are not included. The code also does not include an independent dust species and grain size distribution throughout the disc, making a detailed mineralogy study impossible.

Our model emission profiles are then given by

$F_{\lambda} \sim\left(\sum_{i} \alpha_{i} \kappa_{i}\right) \times\left(\sum_{j} \beta_{j} B_{\lambda}\left(T_{j}\right)\right)$

where $\kappa_{i}$ is the mass absorption coefficient of dust component $i$ and $\alpha_{i}$ gives the fraction of that dust component, $B_{\lambda}\left(T_{j}\right)$ denotes the Planck function at temperature $T_{j}$ and $\beta_{j}$ the fraction of dust in that given temperature. The temperature of dust grains will depend on grain size and grain shape, as well as the distance to the central star and the integrated line-of-sight opacity from the stellar surface to the grains. This can only be calculated using full radiative transfer, so we assume all grains to have the same dust temperatures, irrespective of size and shape.

In this modelling the continuum is given as a sum of Planck functions and we take it as another dust component with a constant mass absorption coefficient. To keep the number of free parameters in our fitting routine reasonable, we allow only two different dust temperatures and continuum temperatures (between 100 and $1000 \mathrm{~K}$ ), with different ratios. A fit with three temperatures was also tested and proved only a minor improvement. The total number of fit parameters in the model is thus 15 , with contributions of four silicate species, where we use two grain sizes, the continuum mass absorption coefficient, two dust and continuum temperatures which each their relative fractions.

To fit the spectra we minimise the reduced $\chi^{2}$ of the full SPITZER spectrum, extended with the IRAS $60 \mu \mathrm{m}$ flux point to constrain the continuum at larger wavelengths, given by

$\chi^{2}=\frac{1}{N-M} \sum_{i=1}^{N}\left|\frac{F^{\text {model }}\left(\lambda_{i}\right)-F^{\text {observed }}\left(\lambda_{i}\right)}{\sigma_{i}}\right|^{2}$,

with $N$ the number of wavelength points $\lambda_{i}, M$ the number of fit parameters and $\sigma_{i}$ the absolute error on the observed flux at wavelength $\lambda_{i}$. The errors $\sigma_{i}$ represent the statistical noise on the spectra. They are generated so that the errors are proportional to the square-root of the flux and they are scaled in such a way that the best fit has a reduced $\chi^{2}$ of approximately 1 .

The errors on the fit parameters are calculated using a MonteCarlo simulation. We randomly add Gaussian noise with a distribution of width $\sigma_{i}$ at each wavelength point. This generates 100 synthetic spectra, all consistent with our data, on which we perform the same fitting procedure. This results in slightly different fit parameters for which we calculate the mean, our best-fit value, and the standard deviation. The uncertainty on the mass absorption coefficients are not taken into account in the $\chi^{2}$-minimalisation.

We find that the mass absorption coefficient of small and large amorphous grains are quite similar, this degeneracy could introduce a large error on the derived fractions for small-large amorphous grains.

\subsection{Results}

We tested both the DHS and the GRF dust approximations in the fitting routine, where the GRF approximation proved a far stronger match. In order to test for the presence of Fe-poor amorphous dust, as postulated in Sect. 5.1, we perform the fitting both with pure Mg-rich amorphous silicates ( $x=1$, see Sect. 5.1) and with the more standard $\mathrm{Mg}-\mathrm{Fe}$ amorphous silicate dust $(x=0.5)$.
When using $0.1 \mu \mathrm{m}$ and $2.0 \mu \mathrm{m}$ grain sizes, we find that nearly $80 \%$ of the dust resides in the $2.0 \mu \mathrm{m}$ grains. The amount of grain growth in crystalline material versus amorphous material is plotted in Fig. 7. Almost all crystalline grains seem to be large, with a fraction of large grains in crystalline component higher than 0.7. For the amorphous grains most sources have fractions between 0.3 and 0.9 . No correlation between grain growth in crystalline and amorphous grains is seen. An efficient removal of the smallest grains must have occurred in these discs or grain growth was the dominant factor to reduce the number of small grains at grain formation.

Because of this lack of small grains, as a next step we performed a fitting using grain sizes of $2.0 \mu \mathrm{m}$ and $4.0 \mu \mathrm{m}$. This resulted in a better fit for $17 / 21$ stars, giving slightly better values for $\chi^{2}$. For EP Lyr and HD 52961 the quality of the fit decreased considerably. This was to be expected, since the strong narrow crystalline features in these stars already indicated the presence of small grains.

In general the fit with the $x=1.0 \mathrm{Mg}$-rich amorphous silicates proved the best, be it only a minor improvement. The observed trends in the fit parameters and stellar characteristics do not change significantly between the fits when using $x=1.0$ or $x=0.5$ amorphous dust. Of our sample stars, 6 stars show a better fit using the $x=0.5$ amorphous silicate dust.

The best model spectra, overplotted with the observed spectra are shown in Figs. 6 and B.8-B.10. The resulting values of the best fit parameters are given in Tables A.1 and A.2. The quality of our fitting is generally very good, $70 \%$ of stars have a $\chi^{2}<5$.

Some trends can be observed (e.g. Figs. 6 and B.8-B.10). As already explained in Sect. 6, the shape of the $33.6 \mu \mathrm{m}$ feature is not well reproduced, although the strength is well modelled. Also the $29 \mu \mathrm{m}$ feature seems to be more prominent in the observed spectra, than in the model fits. The $19 \mu \mathrm{m}$ feature is slightly overestimated in the model spectra, while the neighbouring $23 \mu \mathrm{m}$ feature is slightly underestimated. This discrepancy could be due to a data reduction effect, since in this region there can be a bad overlap between the short and long SPITZER-IRS high-resolution bands.

The clear outliers in our modelling are EP Lyr and HD 52961 with very high values of $\chi^{2}$. These stars have unusual features in their observed spectra, including strong narrow features and $\mathrm{CO}_{2}$ emission lines. A detailed study of these outliers will be given in a follow-up article.

Nearly all sources show the presence of both hot and cool dust, both in dust temperature as in continuum temperature. As seen in Table A.1, dust temperatures can differ strongly from continuum temperatures.

\subsection{Correlations}

To gain insight in the dust formation process and evolution in the circumstellar environment of our sample stars we look for trends in the derived fit parameters and correlations with dust and stellar parameters.

\subsubsection{Mineralogy correlations}

In Fig. 8 the mass fraction in large grains is plotted against the mass fraction in crystalline grains. Mass fractions are calculated as fractions of the total dust mass, excluding the dust responsible for the continuum. Note that small grains now indicate the $2.0 \mu \mathrm{m}$ grain sizes and large grains the $4.0 \mu \mathrm{m}$ grains. 

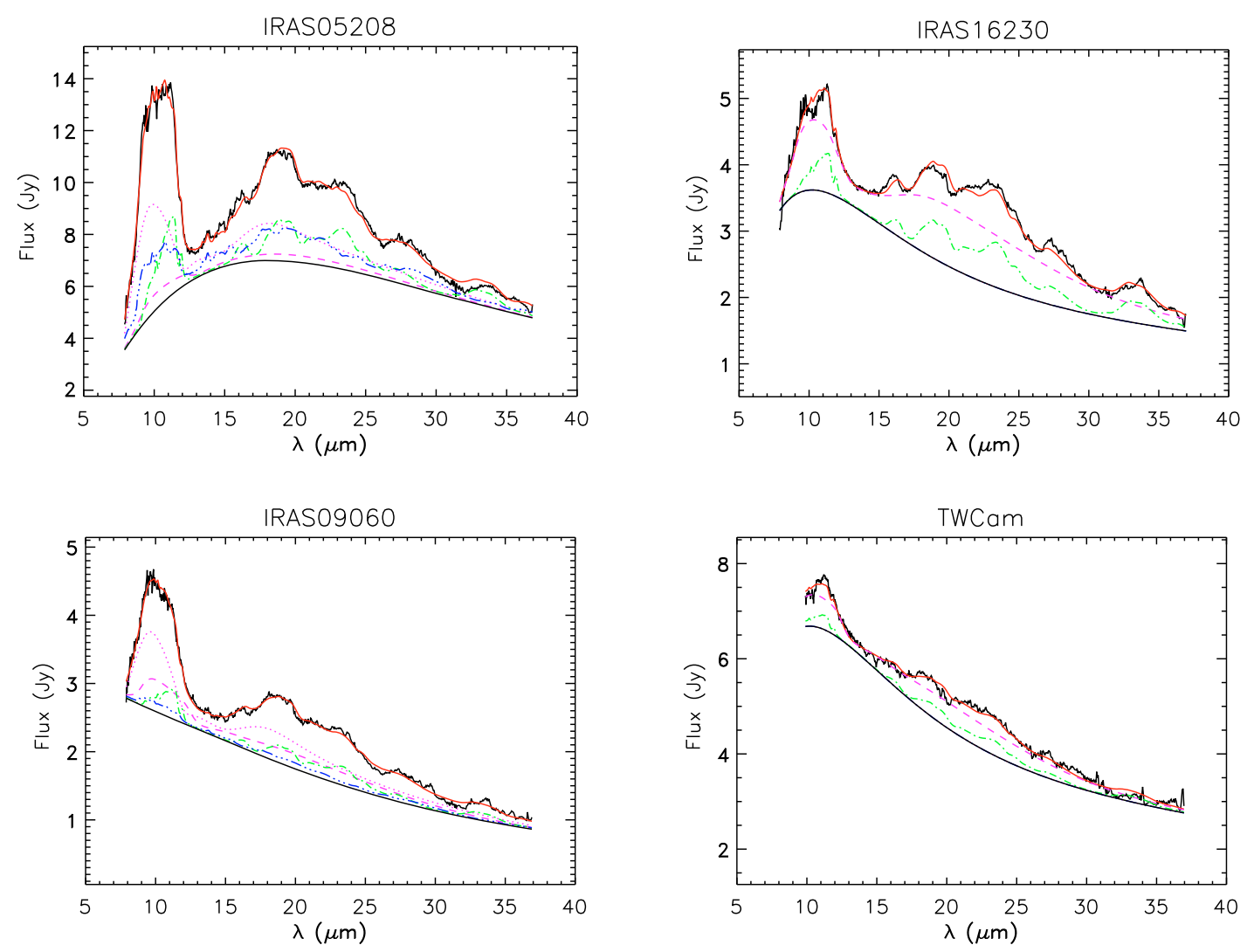

Fig. 6. Best model fits for some of our sample stars, showing the contribution of the different dust species. Best fits for all sample stars are given in Figs. B.8-B.10. The observed spectrum (black curve) is plotted together with the best model fit (red curve) and the continuum (black solid line). Forsterite is plotted in dash-dot lines (green) and enstatite in dash-dot-dotted lines (blue). Small amorphous grains $(2.0 \mu \mathrm{m})$ are plotted as dotted lines (magenta) and large amorphous grains $(4.0 \mu \mathrm{m})$ as dashed lines (magenta).

$85 \%$ of our sources show a mass fraction in large grains above 0.5 . None of the sources have a mass fraction in large grains below 0.25 . Most sources have a mass fraction in crystalline grains between 0.1 and 0.6 , strongly centred around 0.3 . There is one clear outlier: IRAS 10174 with a degree of crystallinity of 0.05 . This source shows the more standard ISM profile, dominated by amorphous grains. No clear correlation can be found.

The fraction of large grains in the crystalline component has values between 0.25 and 0.7 , while the fraction of large grains in the amorphous component has values ranging from 0.3 till 0.95 . Grain growth appears to be more efficient in the amorphous dust component. No correlation between grain growth in crystalline and amorphous grains is seen however.

The fraction of enstatite grains in the crystalline component lies between 0.1 and 0.5 , showing forsterite to be the dominant crystalline species. No correlation between the enstatite fraction and crystallinity can be found.

In Fig. 9 we plot the continuum to dust luminosity ratio of our observed spectra against the mass fraction in large and crystalline grains. A correlation can be found between the continuum/dust ratio and the mass fraction in large grains. Sources with a high fraction of large grains show a high value for the continuum/dust ratio. This could be expected since larger grains show less prominent emission features and have a larger continuum contribution. A high value for the continuum/dust ratio also indicates the presence of even larger grains in the disc. This indicates that the abundance of these larger grains correlate with the abundance of the $4 \mu \mathrm{m}$ grains, and thus that the size distribution continues beyond $4 \mu \mathrm{m}$ grains.

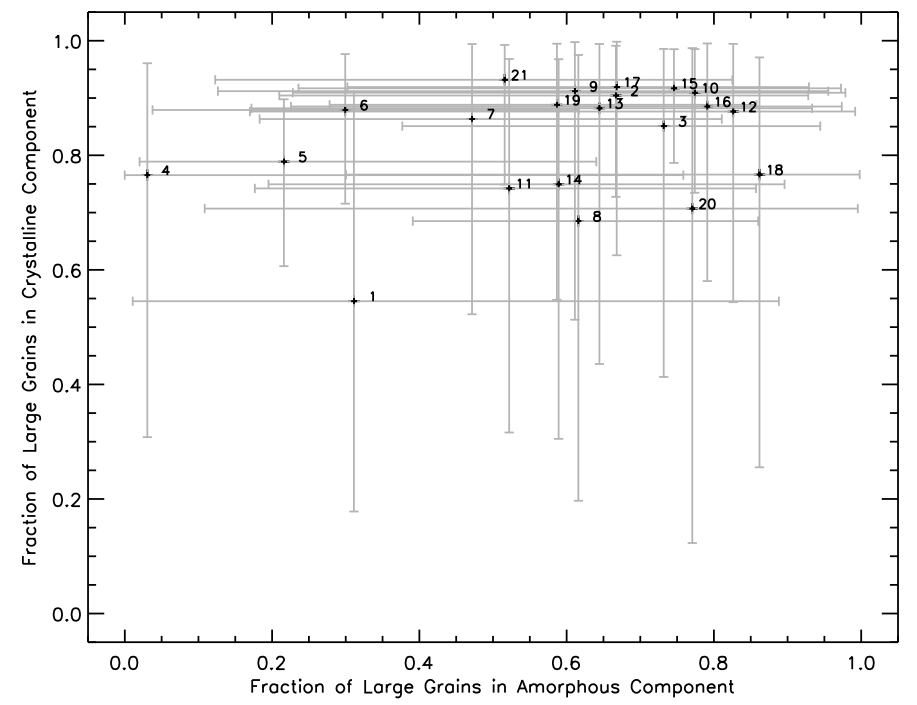

Fig. 7. The mass fraction of large grains in the amorphous component versus the mass fraction of large grains in the crystalline component, using the fitting with grain sizes of $0.1 \mu \mathrm{m}$ and $2.0 \mu \mathrm{m}$. Crystalline grains are almost completely made up from large $2.0 \mu \mathrm{m}$ grains.

\subsubsection{Central star correlations}

By comparing the derived fit parameters with stellar characteristics, like $T_{\mathrm{eff}}, L_{\mathrm{IR}} / L_{*}$ and the orbital period, we can investigate possible evolutionary trends in our sample. However, no such correlation between fit parameters and stellar parameters is 


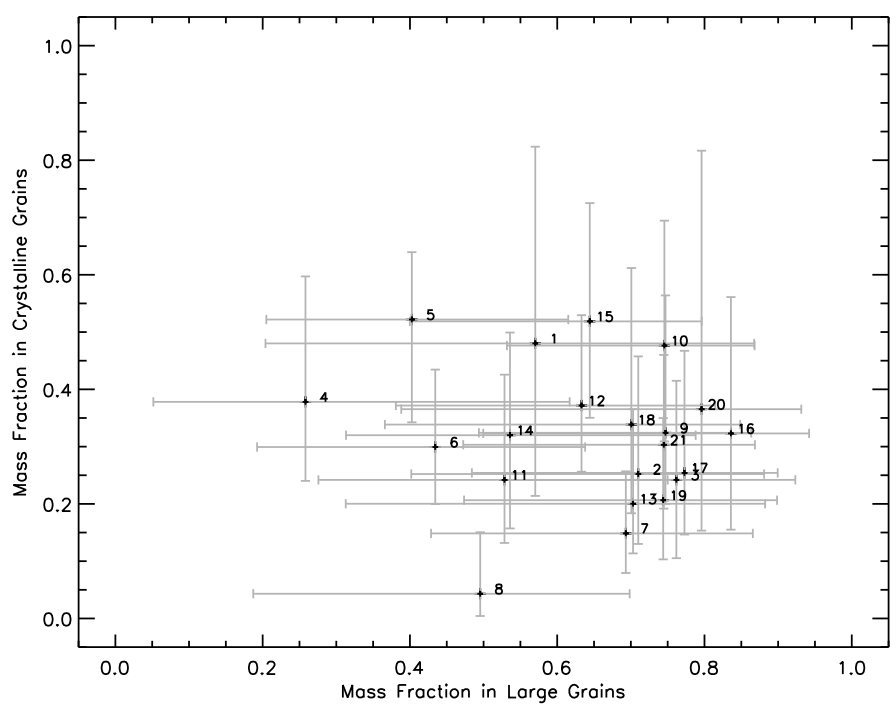

Fig. 8. The mass fraction in large grains plotted against the mass fraction in crystalline grains, as derived from our best fit parameters. A high degree of crystallinity is found.

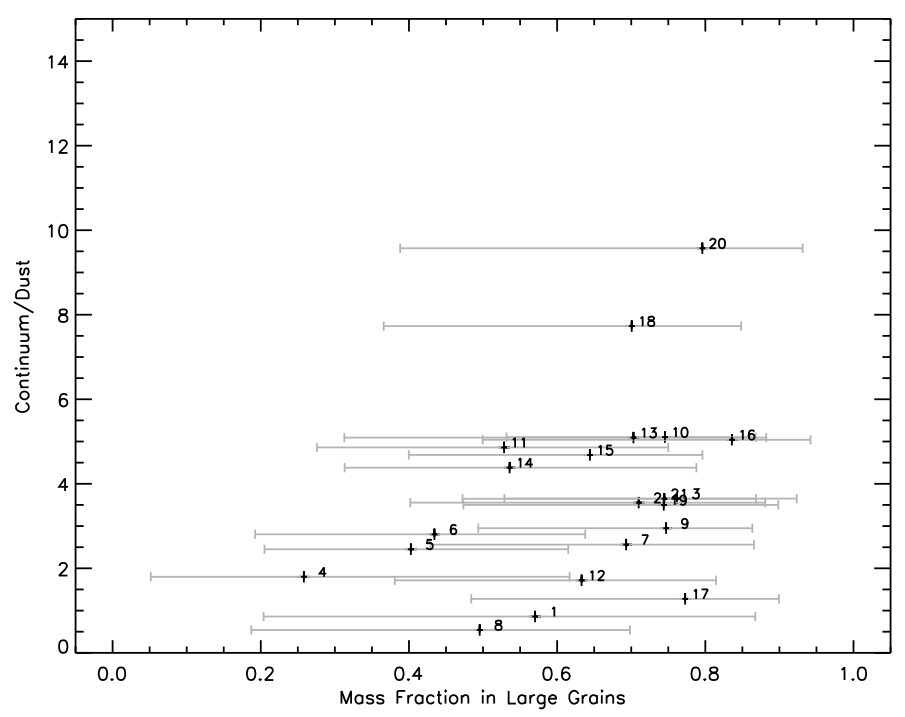

Fig. 9. The continuum to dust ratio of the observed spectra plotted against the mass fraction on large grains.

found. This was also seen in the SEDs (Sect. 4), where nearly all stars show a similar SED, irrespective of the central star. Our results do not show an obvious evolutionary trend in the mineralogy of our sample stars.

\section{Discussion}

The mineralogy of our sample stars show that the dust is purely O-rich. Amorphous and crystalline silicate dust species prevail and no features of a C-rich component are found, except the PAH emission feature seen in EP Lyr.

This is remarkable since some of our sample stars are thought to have initial masses which would make them evolve to carbon stars on the AGB on single-star evolutionary tracks. The lack of third dredge-up is also seen in the two objects with clear $\mathrm{CO}_{2}$ gas emission lines. EP Lyr has a ${ }^{12} \mathrm{C} /{ }^{13} \mathrm{C}$ ratio of $\approx 9$ (Gonzalez et al. 1997b), illustrating that ${ }^{12} \mathrm{C}$ is not enriched during the preceding AGB evolution. The internal chemical evolution of our sources seems to have been cut short by binary interaction processes.

EP Lyr is the only star in our sample which also has a mixed chemistry, and strongly resembles HD 44179. Like HD 44179, EP Lyr also is strongly depleted (Gonzalez et al. 1997b). PAH emission features dominate the spectrum till $20 \mu \mathrm{m}$, at larger wavelengths crystalline silicates start to dominate. The observed broad and asymmetric emission feature at $8.2 \mu \mathrm{m}$ is very similar to the "class C" objects as discussed in Peeters et al. (2002). The famous "Egg Nebula" also shows this "class C" $8.2 \mu \mathrm{m}$ emission feature. EP Lyr, together with HD 52961, will be studied in detail in a future contribution.

Some binary post-AGB stars with oxygen-rich discs are known that did evolve to carbon stars, like HD 44179, which has a large carbon-rich resolved nebula. These stars show infrared spectra which are indicative for mixed chemistries, with features of both oxygen-rich and carbon-rich species. The most likely scenerio for this is that the formation of the O-rich disc antedated the C-rich transition of the central star. Whether other of our sample stars will also undergo this evolution is still unknown.

Our full spectral fitting indicates a high degree of dust grain processing. The dust seems to consist of considerably large grains, with grain sizes larger than $2 \mu \mathrm{m}$. An efficient removal of small grains must have occurred in the discs. The dust shape is highly irregular, showing that Mie theory is not applicable for the dust in these discs. The spectra of nearly all stars show a high degree of crystallinity, where $\mathrm{Mg}$-rich end members of olivine and pyroxene silicates dominate. The dust condensation sequence of dust in winds of oxygen-rich AGB stars predicts the formation of aluminium- or calcium-rich dust grains, like corundum $\left(\mathrm{Al}_{2} \mathrm{O}_{3}\right)$, spinel $\left(\mathrm{MgAl}_{2} \mathrm{O}_{4}\right)$ or anorthite $\left(\mathrm{CaAl}_{2} \mathrm{Si}_{2} \mathrm{O}_{8}\right)$ (Tielens et al. 1997; Cami 2002). There is however no evidence for the presence of these dust species.

Most features are well reproduced and only the 14.7 and $32.5 \mu \mathrm{m}$ features remain unidentified (Sect. B.1). Diopside $\left(\mathrm{CaMgSiO}_{3}\right)$ could be a possible candidate (which has a feature around $14.7 \mu \mathrm{m}$ and a weak feature around $32.1 \mu \mathrm{m}$ ). However, for diopside only mass absorption coefficients for very small grains are available, so we cannot include it in the fitting procedure in a homogeneous way.

The dust is highly magnesium-rich, leaving a large fraction of iron unaccounted for. Previous studies (Gielen et al. 2007) suggest the iron may be locked in the form of metallic iron. Photospheric depletion in iron, which we detect in our sample stars (Maas et al. 2002; Van Winckel et al. 1998), can be understood when the iron is locked up in the circumstellar dust (Waters et al. 1992). The lack of iron in the detected silicates is therefore surprising. If both the crystalline and amorphous silicates are devoid of iron, this could mean that iron is stored in metallic iron or iron-oxide (Sofia et al. 2006). Metallic iron has no distinct features but still makes a significant contribution in opacity, especially at shorter wavelengths, making it very hard to detect directly.

In our fitting method, for both dust temperatures equal silicate fractions are used. This seems to fit features indicative of both hot and cool crystalline dust, meaning we do not have a strong radial gradient in crystallinity throughout the disc. The inner regions of the discs, with temperatures above the annealing temperature, are expected to be fully crystalline. The presence of a considerable amount of cool crystalline grains implies thus that we have strong turbulent mixing in the disc or that the crystalline grains were already abundant at disc formation. It is interesting to note that the very young circumbinary disc in the evolved binary SS Lep (Verhoelst et al. 2007), which may be 
formed in a similar process as the discs around our post-AGB sources, is dominated by small and large amorphous grains. The crystalline component is very small (Schütz et al. 2005). This would indicate that the crystallisation process happens during disc evolution and is not already present at formation.

The evolution of these discs is still unknown. Our analysis shows no clear correlation between dust parameters and any fundamental parameter $\left(T_{\text {eff }}\right.$, orbital period) of the central star. In a recent study of Chesneau et al. (2007), a compact dusty disc was discovered in "the Ant", a well studied bipolar planetary nebula. Interferometric MIDI observations provided evidence for a flat, nearly edge-on disc, primarily composed of amorphous silicates. This is in contrast with the high crystallinity observed in the discs around binary post-AGB stars, suggesting that the disc in the Ant is relatively young. Whether there is a link between this disc and the post-AGB discs remains unclear.

Some degeneracy is present in our spectral fits. We performed the spectral fitting both with pure Mg-rich amorphous silicates $(x=1)$ and with the more standard Mg-Fe amorphous silicate dust $(x=0.5)$. Both models often have similar $\chi^{2}$ values and the best model strongly varies from star to star. Some stars also show equally well-fitting models, when using $0.1-2.0 \mu \mathrm{m}$ or $2.0-4.0 \mu \mathrm{m}$ grain sizes.

\subsection{Comparison with young stellar objects}

The mineralogy of the observed spectra shows a striking resemblance to the infrared spectra of young stellar objects, like Herbig Ae/Be stars (Lisse et al. 2007; van Boekel et al. 2005) or T Tauri stars (Watson et al. 2007), and primitive comets such as Hale-Bopp (Lisse et al. 2007; Bouwman et al. 2003; Min et al. 2005b). There also, amorphous silicates and Mg-rich crystalline features prevail. The dusty disc is the relic of the star formation process so both silicates together with a carbon-rich component in the form of PAH emission are often detected, depending on the disc geometry (Acke \& van den Ancker 2004).

We compare our findings with the ones discussed in van Boekel et al. (2005). They present spectroscopic observations of a large sample of Herbig Ae/Be stars in the $10 \mu \mathrm{m}$ region. Similar studies on young stellar objects have also been done by e.g. Bouwman et al. (2001). One has to be careful comparing studies, since our study includes a far larger wavelength range, and an exact comparison is thus not possible.

The degree of crystallinity found in the Herbig Ae/Be stars is clearly smaller than for our sample of post-AGB binaries. The Herbig stars all show a degree of crystallinity below 0.35 , whereas our stars have significantly higher values. van Boekel et al. (2005) use both small $(0.1 \mu \mathrm{m})$ and large $(1.5 \mu \mathrm{m})$ grains to fit the observed emission features and find that most sources have a mass fraction in large grains of more than $80 \%$.

For both young and evolved objects a substantial removal of the smallest grains has occurred, but seems to be more efficient in the discs around the evolved stars. A similar physical process might be responsible for the observed grain size distribution in both cases, like aggregation of small grains or the removal of small grains by radiation pressure. Since several processes occur in the formation of the disc it could also be that silicate grains in the post-AGB disc are formed at large grain sizes. These large grains could be broken up by grain interactions in the disc, producing a small fraction of small grains.

van Boekel et al. (2005) found a clear correlation between the crystallinity and the dominance of enstatite or forsterite in these grains. For sources with a high degree of crystallinity most crystalline grains appear to be in the form of enstatite, while for sources with a low crystallinity, forsterite seems to be the dominant species. For all our sample stars forsterite is the dominant species, despite the high crystallinity factors. This difference could relate to the initial dust species when the disc is formed. For discs around YSO, which are formed from the ISM, the abundant dust species is likely amorphous olivine. Forsterite is expected to be the dominant species formed by thermal annealing, while enstatite is expected to be the dominant species formed by chemical equilibrium processes in most of the inner disc. Our discs seem to prefer the formation of forsterite, but the initial dust species is not known. The innermost disc regions are hot enough to crystallise dust, but the very high degree of crystallinity seems to point to the presence of another crystallisation processes, possibly at disc formation.

\section{Conclusions}

We present high-resolution TIMMI2 and SPITZER infrared spectra of 21 binary post-AGB stars surrounded by a stable Keplerian disc. We summarise our main conclusions:

- Almost all discs display only O-rich spectral signatures. The noticeable exception is EP Lyr, which shows a very similar spectrum as the central star of the Red Rectangle. PAH emission features dominate the spectrum till $20 \mu \mathrm{m}$, at larger wavelengths crystalline silicates start to dominate.

- Our mineralogy study indicates the dominance of Mg-rich amorphous and crystalline silicate dust in the disc. The high crystallinity and the large fraction of large grains, as deduced from our full spectral fitting, show strong dust grain processing in the discs.

- The temperature estimates from our fitting routine show that a significant fraction of crystalline grains must be cool. This shows that radial mixing is efficient is these discs or indicate a different thermal history at grain formation.

- Trend analysis of our fitting parameters show no clear correlation with stellar characteristics. For the moment it is not clear if and how the observed diversity in observed spectra relates to specific structural elements of the disc, the star and/or the orbits or whether we witness directly an evolutionary change between different sources.

To further improve our understanding of these circumbinary discs, as a next step, we will combine our photometric and spectroscopic data with interferometric measurements. Comparing spatial information from the MIDI and AMBER interferometric instruments with a realistic disc model (Dullemond \& Dominik 2004), constrained by photometric and spectroscopic data, will allow us, not only to study the mineralogy, but also the structure of the discs. So far, interferometric measurements for five of our sample stars have been obtained (Deroo et al. 2006; Deroo 2007).

Probing the dust processing in the discs around evolved objects proves to be an excellent complement to study physics in planet-forming young discs.

Acknowledgements. The authors want to acknowledge: the $1.2 \mathrm{~m}$ Mercator staff as well as the observers from the Instituut voor Sterrenkunde who contributed to the monitoring observations using the Mercator telescope. C.G. acknowledges support of the Fund for Scientific Research of Flanders (FWO) under the grant G.0178.02. and G.0470.07. We also thank Fred Lahuis for his assistance with the SPITZER data reduction. 


\section{References}

Acke, B., \& van den Ancker, M. E. 2004, A\&A, 426, 151

Aden, A. L., \& Kerker, M. 1951, J. Appl. Phys., 22, 1242

Bohren, C. F., Huffman, D. R., \& Kam, Z. 1983, Nature, 306, 625

Bouwman, J., Meeus, G., de Koter, A., et al. 2001, A\&A, 375, 950

Bouwman, J., de Koter, A., Dominik, C., \& Waters, L. B. F. M. 2003, A\&A, 401, 577

Bradley, J. P., Brownlee, D. E., \& Veblen, D. R. 1983, Nature, 301, 473

Bujarrabal, V., Bachiller, R., Alcolea, J., \& Martin-Pintado, J. 1988, A\&A, 206, L17

Bujarrabal, V., Castro-Carrizo, A., Alcolea, J., \& Neri, R. 2005, A\&A, 441, 1031 Bujarrabal, V., Van Winckel, H., Neri, R., et al. 2007, A\&A, 468, L45

Cami, J. 2002, Ph.D. Thesis, University of Amsterdam

Chesneau, O., Lykou, F., Balick, B., et al. 2007, A\&A, 473, L29

Cohen, M., Van Winckel, H., Bond, H. E., \& Gull, T. R. 2004, AJ, 127, 2362

De Ruyter, S., Van Winckel, H., Maas, T., et al. 2006, A\&A, 448, 641

Deroo, P. 2007, Ph.D. Thesis, KULeuven

Deroo, P., Van Winckel, H., Min, M., et al. 2006, A\&A, 450, 181

Deroo, P., Acke, A., Verhoelst, T., et al. 2007a, A\&A, 468

Deroo, P., Van Winckel, H., Verhoelst, T., et al. 2007b, A\&A, 467, 1093

Dorschner, J., Begemann, B., Henning, T., Jaeger, C., \& Mutschke, H. 1995 A\&A, 300, 503

Draine, B. T. 1988 , ApJ, 333, 848

Dullemond, C. P., \& Dominik, C. 2004, A\&A, 417, 159

Fabian, D., Jäger, C., Henning, T., Dorschner, J., \& Mutschke, H. 2000, A\&A, 364,282

Gielen, C., Van Winckel, H., Waters, L. B. F. M., Min, M., \& Dominik, C. 2007, A\&A, 475, 629

Giridhar, S., Rao, N. K., \& Lambert, D. L. 1994, ApJ, 437, 476

Giridhar, S., Lambert, D. L., \& Gonzalez, G. 1998, ApJ, 509, 366

Giridhar, S., Lambert, D. L., \& Gonzalez, G. 2000, ApJ, 531, 521

Gledhill, T. M., Chrysostomou, A., Hough, J. H., \& Yates, J. A. 2001, MNRAS, 322,321

Gonzalez, G., Lambert, D. L., \& Giridhar, S. 1997a, ApJ, 481, 452

Gonzalez, G., Lambert, D. L., \& Giridhar, S. 1997b, ApJ, 479, 427

Grynko, Y., \& Shkuratov, Y. 2003, J. Quant. Spectr. Radiat. Transfer, 78, 319

Henning, T., \& Stognienko, R. 1996, A\&A, 311, 291

Honda, M., Kataza, H., Okamoto, Y. K., et al. 2004, ApJ, 610, L49

Hony, S., Van Kerckhoven, C., Peeters, E., et al. 2001, A\&A, 370, 1030

Houck, J. R., Roellig, T. L., van Cleve, J., et al. 2004, ApJS, 154, 18

Hrivnak, B. J., Kwok, S., \& Volk, K. M. 1989, ApJ, 346, 265

Jaeger, C., Molster, F. J., Dorschner, J., et al. 1998, A\&A, 339, 904

Käufl, H.-U., Sterzik, M. F., Siebenmorgen, R., et al. 2003, in Instrument Design and Performance for Optical/Infrared Ground-based Telescopes, presented at the Society of Photo-Optical Instrumentation Engineers (SPIE) Conference, ed. M. Iye, \& A. F. M. Moorwood, Proc. SPIE, 4841, 117

Kemper, F., Vriend, W. J., \& Tielens, A. G. G. M. 2004, ApJ, 609, 826

Kessler-Silacci, J., Augereau, J.-C., Dullemond, C. P., et al. 2006, ApJ, 639, 275

Kurucz, R. L. 1979, ApJS, 40, 1

Kwok, S., Volk, K. M., \& Hrivnak, B. J. 1989, ApJ, 345, L51

Lahuis, F., et al. 2006, c2d Spectroscopy Explanatory Supplement, Cores to Disks, Spitzer Legacy Team (Pasadena: Spitzer Science Center)

Lisse, C. M., Kraemer, K. E., Nuth, J. A., Li, A., \& Joswiak, D. 2007, Icarus, 187,69

Lloyd Evans, T. 1985, MNRAS, 217, 493

Lloyd Evans, T. 1999, in Asymptotic Giant Branch Stars, ed. T. Le Bertre, A. Lebre, \& C. Waelkens, IAU Symp., 191, 453

Maas, T., Van Winckel, H., \& Waelkens, C. 2002, A\&A, 386, 504
Maas, T., Van Winckel, H., \& Lloyd Evans, T. 2003, in ASP Conf. Ser., ed. R. L. M. Corradi, J. Mikolajewska, \& T. J. Mahoney, 303, 143

Maas, T., Van Winckel, H., \& Lloyd Evans, T. 2005, A\&A, 429, 297

Mastrodemos, N., \& Morris, M. 1999, ApJ, 523, 357

Meixner, M., Gordon, K. D., Indebetouw, R., et al. 2006, AJ, 132, 2268

Men'shchikov, A. B., Schertl, D., Tuthill, P. G., Weigelt, G., \& Yungelson, L. R. 2002, A\&A, 393, 867

Menzies, J. W., \& Whitelock, P. A. 1988, MNRAS, 233, 697

Min, M., Hovenier, J. W., \& de Koter, A. 2003, A\&A, 404, 35

Min, M., Hovenier, J. W., \& de Koter, A. 2005a, A\&A, 432, 909

Min, M., Hovenier, J. W., de Koter, A., Waters, L. B. F. M., \& Dominik, C. 2005b, Icarus, 179, 158

Min, M., Waters, L. B. F. M., de Koter, A., et al. 2007, A\&A, 462, 667

Molster, F. J., Yamamura, I., Waters, L. B. F. M., et al. 1999, Nature, 401, 563

Molster, F. J., Waters, L. B. F. M., \& Tielens, A. G. G. M. 2002a, A\&A, 382, 222

Molster, F. J., Waters, L. B. F. M., Tielens, A. G. G. M., \& Barlow, M. J. 2002b, A\&A, 382, 184

Molster, F. J., Waters, L. B. F. M., Tielens, A. G. G. M., Koike, C., \& Chihara, H. 2002c, A\&A, 382, 241

Oudmaijer, R. D., van der Veen, W. E. C. J., Waters, L. B. F. M., et al. 1992, A\&AS, 96, 625

Peeters, E., Hony, S., Van Kerckhoven, C., et al. 2002, A\&A, 390, 1089

Raveendran, A. V. 1989, MNRAS, 238, 945

Reimann, H.-G., Linz, H., Wagner, R., et al. 2000, in Optical and IR Telescope Instrumentation and Detectors, ed. M. Iye, \& A. F. Moorwood, Proc. SPIE, 4008, 1132

Reyniers, M., \& Van Winckel, H. 2007, A\&A, 463, L1

Roddier, F., Roddier, C., Graves, J. E., \& Northcott, M. J. 1995, ApJ, 443, 249

Savage, B. D., \& Mathis, J. S. 1979, ARA\&A, 17, 73

Schütz, O., Meeus, G., \& Sterzik, M. F. 2005, A\&A, 431, 175

Servoin, J. L., \& Pirou, B. 1973, Phys. Stat. Sol. B, 55, 677

Shkuratov, Y. G., \& Grynko, Y. S. 2005, Icarus, 173, 16

Sofia, U. J., Gordon, K. D., Clayton, G. C., et al. 2006, ApJ, 636, 753

Steenman, H., \& Thé, P. S. 1989, Ap\&SS, 159, 189

Steenman, H., \& Thé, P. S. 1991, Ap\&SS, 184, 9

Szczerba, R., Siódmiak, N., Stasińska, G., \& Borkowski, J. 2007, A\&A, 469, 799

Tielens, A. G. G. M., Waters, L. B. F. M., Molster, F. J., \& Justtanont, K. 1997, Ap\&SS, 255, 415

Toon, O. B., \& Ackerman, T. P. 1981, Appl. Opt., 20, 3657

van Boekel, R., Waters, L. B. F. M., Dominik, C., et al. 2003, A\&A, 400, L21

van Boekel, R., Min, M., Waters, L. B. F. M., et al. 2005, A\&A, 437, 189

Van Kerckhoven, C., Hony, S., Peeters, E., et al. 2000, A\&A, 357, 1013

Van Winckel, H. 2003, ARA\&A, 41, 391

Van Winckel, H. 2007, Baltic Astron., 16, 112

Van Winckel, H., Waelkens, C., \& Waters, L. B. F. M. 1995, A\&A, 293, L25

Van Winckel, H., Waelkens, C., Waters, L. B. F. M., et al. 1998, A\&A, 336, L17

Van Winckel, H., Waelkens, C., Fernie, J. D., \& Waters, L. B. F. M. 1999, A\&A, 343, 202

Verhoelst, T., van Aarle, E., \& Acke, B. 2007, A\&A, 470, L21

Waelkens, C., Waters, L. B. F. M., de Graauw, M. S., et al. 1996, A\&A, 315, L245

Waters, L. B. F. M., Trams, N. R., \& Waelkens, C. 1992, A\&A, 262, L37

Waters, L. B. F. M., Molster, F. J., de Jong, T., et al. 1996, A\&A, 315, L361

Waters, L. B. F. M., Cami, J., de Jong, T., et al. 1998, Nature, 391, 868

Watson, D. M., Leisenring, J. M., Furlan, E., et al. 2007, ArXiv e-prints, 704

Werner, M. W., Roellig, T. L., Low, F. J., et al. 2004, ApJS, 154, 1 
C. Gielen et al.: SPITZER survey of dust grain processing in stable discs around binary post-AGB stars, Online Material p 1

\section{Appendix A: Tables}

Table A.1. Best fit parameters deduced from our full spectral fitting. Listed the $\chi^{2}$, dust and continuum temperatures and their relative fractions.

\begin{tabular}{|c|c|c|c|c|c|c|c|c|}
\hline $\mathrm{N}^{\circ}$ & Name & $\overline{\chi^{2}}$ & $\begin{array}{l}T_{\text {dust1 }} \\
(\mathrm{K})\end{array}$ & $\begin{array}{l}T_{\text {dust2 }} \\
(\mathrm{K})\end{array}$ & $\begin{array}{l}\text { Fraction } \\
T_{\text {dust1 }}-T_{\text {dust2 }}\end{array}$ & $\begin{array}{l}T_{\text {cont1 }} \\
(\mathrm{K})\end{array}$ & $\begin{array}{l}T_{\text {cont2 }} \\
(\mathrm{K})\end{array}$ & $\begin{array}{l}\text { Fraction } \\
T_{\text {cont1 }}-T_{\text {cont2 }} \\
\end{array}$ \\
\hline 1 & EP Lyr & 56.1 & $116_{16}^{97}$ & $237_{37}^{277}$ & $0.80_{0.30}^{0.10}-0.20_{0.10}^{0.30}$ & $195_{100}^{45}$ & $921_{342}^{80}$ & $0.98_{0.02}^{0.01}-0.02_{0.01}^{0.02}$ \\
\hline 2 & HD 131356 & 3.5 & $226_{46}^{269}$ & $928_{187}^{73}$ & $0.80_{0.50}^{0.10}-0.20_{0.10}^{0.50}$ & $195_{104}^{12}$ & $534_{50}^{95}$ & $0.92_{0.02}^{0.03}-0.08_{0.03}^{0.02}$ \\
\hline 3 & HD 213985 & 4.1 & $185_{87}^{45}$ & $926_{333}^{74}$ & $0.80_{0.40}^{0.10}-0.20_{0.10}^{0.40}$ & $187_{91}^{13}$ & $838_{162}^{73}$ & $0.98_{0.00}^{0.01}-0.02_{0.01}^{0.00}$ \\
\hline 4 & HD 52961 & 72.2 & $199_{140}^{10}$ & $845_{106}^{121}$ & $0.90_{0.70}^{0.00}-0.10_{0.00}^{0.70}$ & $109_{9}^{497}$ & $998_{139}^{2}$ & $0.99_{0.03}^{0.00}-0.01_{0.00}^{0.03}$ \\
\hline 5 & IRAS 05208 & 4.5 & $291_{101}^{176}$ & $904_{124}^{97}$ & $0.80_{0.30}^{0.10}-0.20_{0.10}^{0.30}$ & $177_{79}^{30}$ & $374_{76}^{26}$ & $0.84_{0.03}^{0.02}-0.16_{0.02}^{0.03}$ \\
\hline 6 & IRAS 09060 & 3.6 & $206_{6}^{176}$ & $755_{163}^{140}$ & $0.90_{0.30}^{0.00}-0.10_{0.00}^{0.30}$ & $225_{122}^{86}$ & $805_{198}^{162}$ & $0.93_{0.02}^{0.02}-0.07_{0.02}^{0.02}$ \\
\hline 7 & IRAS 09144 & 6.1 & $212_{24}^{91}$ & $535_{78}^{137}$ & $0.90_{0.20}^{0.00}-0.10_{0.00}^{0.20}$ & $196_{105}^{16}$ & $767_{89}^{89}$ & $0.94_{0.02}^{0.02}-0.06_{0.02}^{0.02}$ \\
\hline 8 & IRAS 10174 & 13.9 & $234_{125}^{72}$ & $378_{79}^{47}$ & $0.70_{0.40}^{0.20}-0.30_{0.20}^{0.40}$ & $125_{25}^{76}$ & $322_{22}^{95}$ & $0.96_{0.04}^{0.02}-0.04_{0.02}^{0.04}$ \\
\hline 9 & IRAS 16230 & 4.9 & $210_{22}^{143}$ & $507_{74}^{182}$ & $0.90_{0.20}^{0.00}-0.10_{0.00}^{0.20}$ & $151_{51}^{154}$ & $592_{93}^{286}$ & $0.94_{0.03}^{0.01}-0.06_{0.01}^{0.03}$ \\
\hline 10 & IRAS 17038 & 2.9 & $250_{69}^{140}$ & $854_{192}^{120}$ & $0.80_{0.20}^{0.10}-0.20_{0.10}^{0.20}$ & $198_{139}^{2}$ & $563_{85}^{84}$ & $0.96_{0.02}^{0.01}-0.04_{0.01}^{0.02}$ \\
\hline 11 & IRAS 17243 & 2.3 & $210_{38}^{110}$ & $441_{79}^{89}$ & $0.80_{0.50}^{0.10}-0.20_{0.10}^{0.50}$ & $202_{15}^{113}$ & $633_{39}^{168}$ & $0.91_{0.01}^{0.03}-0.09_{0.03}^{0.01}$ \\
\hline 12 & IRAS 19125 & 3.9 & $102_{2}^{36}$ & $204_{4}^{277}$ & $0.90_{0.10}^{0.00}-0.10_{0.00}^{0.10}$ & $321_{138}^{169}$ & $702_{84}^{126}$ & $0.84_{0.04}^{0.04}-0.16_{0.04}^{0.03}$ \\
\hline 13 & IRAS 19157 & 5.5 & $199_{111}^{26}$ & $609_{159}^{176}$ & $0.90_{0.40}^{0.00}-0.10_{0.00}^{0.40}$ & $186_{89}^{140}$ & $689_{137}^{85}$ & $0.96_{0.02}^{0.01}-0.04_{0.01}^{0.02}$ \\
\hline 14 & IRAS 20056 & 3.8 & $118_{18}^{84}$ & $219_{19}^{83}$ & $0.60_{0.60}^{0.20}-0.40_{0.20}^{0.60}$ & $238_{51}^{87}$ & $658_{72}^{119}$ & $0.88_{0.04}^{0.01}-0.12_{0.01}^{0.01}$ \\
\hline 15 & RU Cen & 3.4 & $233_{67}^{87}$ & $517_{115}^{200}$ & $0.80_{0.40}^{0.10}-0.20_{0.10}^{0.40}$ & $199_{0}^{1}$ & $545_{125}^{77}$ & $0.98_{0.02}^{0.01}-0.02_{0.01}^{0.01}$ \\
\hline 16 & SAO 173329 & 3.1 & $205_{36}^{160}$ & $764_{133}^{115}$ & $0.80_{0.50}^{0.10}-0.20_{0.10}^{0.50}$ & $195_{106}^{5}$ & $581_{100}^{23}$ & $0.93_{0.02}^{0.01}-0.07_{0.02}^{0.01}$ \\
\hline 17 & ST Pup & 8.4 & $207_{13}^{99}$ & $480_{86}^{86}$ & $0.80_{0.20}^{0.10}-0.20_{0.10}^{0.20}$ & $207_{13}^{99}$ & $524_{57}^{180}$ & $0.95_{0.03}^{0.02}-0.05_{0.03}^{0.01}$ \\
\hline 18 & SU Gem & 1.8 & $241_{98}^{336}$ & $629_{184}^{284}$ & $0.80_{0.30}^{0.10}-0.20_{0.10}^{0.30}$ & $177_{79}^{23}$ & $763_{192}^{137}$ & $0.95_{0.02}^{0.02}-0.05_{0.02}^{0.02}$ \\
\hline 19 & SX Cen & 4.3 & $225_{44}^{202}$ & $918_{183}^{83}$ & $0.80_{0.30}^{0.10}-0.20_{0.10}^{0.30}$ & $191_{96}^{9}$ & $659_{80}^{952}$ & $0.94_{0.02}^{0.02}-0.06_{0.02}^{0.02}$ \\
\hline 20 & TW Cam & 2.3 & $216_{82}^{123}$ & $390_{171}^{107}$ & $0.70_{0.40}^{0.30}-0.30_{0.20}^{0.40}$ & $131_{31}^{321}$ & $546_{46}^{205}$ & $0.95_{0.02}^{0.02}-0.05_{0.01}^{0.02}$ \\
\hline 21 & UY CMa & 2.9 & $205_{12}^{104}$ & $761_{113}^{131}$ & $0.90_{0.00}^{0.00}-0.10_{0.00}^{0.00}$ & $203_{3}^{119}$ & $511_{11}^{126}$ & $0.84_{0.02}^{0.03}-0.16_{0.03}^{0.02}$ \\
\hline
\end{tabular}

Table A.2. Best fit parameters deduced from our full spectral fitting. The abundances of small $(2.0 \mu \mathrm{m})$ and large $(4.0 \mu \mathrm{m})$ grains of the various dust species are given as fractions of the total mass, excluding the dust responsible for the continuum emission. The last column gives the continuum flux contribution, listed as a percentage of the total integrated flux over the full wavelength range.

\begin{tabular}{|c|c|c|c|c|c|c|}
\hline $\mathrm{N}^{\circ}$ & Name & $\begin{array}{c}\text { Olivine } \\
\text { Small-Large }\end{array}$ & $\begin{array}{c}\text { Pyroxene } \\
\text { Small-Large }\end{array}$ & $\begin{array}{c}\text { Forsterite } \\
\text { Small-Large }\end{array}$ & $\begin{array}{c}\text { Enstatite } \\
\text { Small-Large }\end{array}$ & Continuum \\
\hline 1 & EP Lyr & $0.17_{0.17}^{7.59}-5.61_{5.61}^{46.82}$ & $19.22_{17.10}^{34.23}-26.98_{24.42}^{40.96}$ & $20.42_{14.25}^{32.78}-7.80_{7.46}^{27.63}$ & $3.17_{3.13}^{26.21}-16.63_{14.58}^{29.16}$ & $58.26_{8.63}^{8.69}$ \\
\hline 2 & HD 131356 & $1.58_{1.57}^{25.29}-29.75_{21.09}^{15.77}$ & $12.99_{11.34}^{18.61}-30.47_{25.45}^{21.47}$ & $13.93_{7.29}^{10.91}-3.63_{3.57}^{18.73}$ & $0.49_{0.49}^{5.85}-7.17_{6.82}^{17.49}$ & $77.78_{2.72}^{3.03}$ \\
\hline 3 & HD 213985 & $1.51_{1.52}^{21.49}-28.81_{21.16}^{14.62}$ & $14.12_{12.08}^{15.94}-31.37_{22.90}^{27.40}$ & $7.64_{5.58}^{10.20}-8.50_{7.60}^{17.33}$ & $0.55_{0.56}^{6.53}-7.50_{6.31}^{10.07}$ & $75.05_{5.53}^{3.51}$ \\
\hline 4 & HD 52961 & $0.21_{0.21}^{15.75}-0.59_{0.59}^{45.99}$ & $54.40_{30.88}^{16.42}-7.00_{7.04}^{55.05}$ & $17.33_{12.99}^{15.22}-11.53_{10.64}^{22.97}$ & $2.26_{2.26}^{21.87}-6.69_{6.49}^{32.31}$ & $64.91_{6.83}^{4.75}$ \\
\hline 5 & IRAS 05208 & $1.91_{1.90}^{16.88}-7.39_{6.59}^{12.81}$ & $29.09_{13.86}^{11.19}-9.42_{8.97}^{25.21}$ & $23.04_{10.05}^{8.08}-2.70_{2.69}^{17.04}$ & $5.70_{5.25}^{10.35}-20.75_{15.60}^{13.58}$ & $67.64_{4.27}^{2.74}$ \\
\hline 6 & IRAS 09060 & $7.20_{7.04}^{24.66}-19.67_{17.20}^{16.68}$ & $34.08_{18.50}^{13.73}-9.12_{9.02}^{32.11}$ & $14.03_{6.33}^{7.88}-5.10_{4.97}^{15.99}$ & $1.25_{1.24}^{10.01}-9.55_{7.17}^{10.30}$ & $71.99_{3.46}^{2.60}$ \\
\hline 7 & IRAS 09144 & $1.05_{1.06}^{23.75}-32.86_{19.81}^{15.70}$ & $20.98_{14.76}^{21.45}-30.25_{23.38}^{27.10}$ & $8.39_{5.42}^{8.05}-2.96_{2.89}^{10.25}$ & $0.26_{0.26}^{6.45}-3.23_{3.18}^{10.74}$ & $72.99_{3.25}^{3.40}$ \\
\hline 8 & IRAS 10174 & $21.58_{18.72}^{27.48}-28.94_{24.31}^{20.10}$ & $27.36_{15.16}^{15.22}-17.81_{15.55}^{12.52}$ & $0.85_{0.84}^{6.88}-1.70_{1.66}^{9.53}$ & $0.68_{0.68}^{8.72}-1.09_{1.09}^{7.09}$ & $35.70_{5.72}^{6.39}$ \\
\hline 9 & IRAS 16230 & $3.13_{3.13}^{30.37}-43.75_{30.34}^{19.08}$ & $4.15_{3.96}^{12.04}-16.56_{15.24}^{18.26}$ & $18.02_{8.89}^{11.45}-9.20_{7.55}^{22.74}$ & $0.00_{0.00}^{0.00}-5.19_{4.95}^{20.91}$ & $77.27_{2.66}^{4.17}$ \\
\hline 10 & IRAS 17038 & $0.79_{0.80}^{55.99}-22.98_{17.86}^{19.89}$ & $4.02_{3.95}^{13.05}-24.56_{17.78}^{18.20}$ & $19.33_{11.24}^{11.07}-10.25_{9.80}^{23.93}$ & $1.31_{1.31}^{8.97}-16.76_{12.88}^{18.14}$ & $81.62_{2.78}^{2.00}$ \\
\hline 11 & IRAS 17243 & $8.62_{8.41}^{13.13}-31.99_{24.71}^{17.50}$ & $22.07_{13.70}^{16.69}-13.14_{12.05}^{28.77}$ & $16.36_{8.44}^{9.05}-1.27_{1.25}^{15.32}$ & $0.12_{0.12}^{4.41}-6.43_{5.36}^{13.45}$ & $83.09_{2.53}^{1.90}$ \\
\hline 12 & IRAS 19125 & $10.68_{9.79}^{24.63}-9.13_{8.38}^{19.94}$ & $15.45_{11.32}^{21.11}-27.57_{21.85}^{15.18}$ & $9.04_{5.23}^{6.52}-10.35_{6.74}^{12.02}$ & $1.54_{1.53}^{11.44}-16.24_{9.74}^{11.04}$ & $72.70_{3.23}^{3.73}$ \\
\hline 13 & IRAS 19157 & $9.14_{9.12}^{40.25}-48.99_{36.60}^{20.74}$ & $9.67_{7.79}^{13.47}-12.17_{11.69}^{25.87}$ & $10.71_{7.70}^{9.97}-5.13_{5.00}^{16.740}$ & $0.18_{0.18}^{8.99}-4.02_{3.78}^{14.16}$ & $80.17_{4.80}^{2.85}$ \\
\hline 14 & IRAS 20056 & $6.12_{6.08}^{27.24}-20.84_{17.18}^{23.73}$ & $27.05_{19.61}^{20.02}-14.01_{13.25}^{35.34}$ & $11.59_{7.25}^{9.40}-5.04_{4.86}^{15.70}$ & $1.67_{1.67}^{10.66}-13.69_{11.05}^{15.29}$ & $81.36_{3.32}^{2.70}$ \\
\hline 15 & RU Cen & $0.98_{0.98}^{160}-22.37_{17.52}^{16.10}$ & $5.74_{5.32}^{11.49}-19.03_{16.49}^{17.47}$ & $27.79_{11.46}^{14.30}-10.31_{8.72}^{4.40}$ & $1.07_{1.07}^{12.34}-12.70_{10.10}^{17.09}$ & $80.23_{3.34}^{2.82}$ \\
\hline 16 & SAO 173329 & $1.07_{1.07}^{23.25}-41.52_{28.71}^{18.10}$ & $4.19_{4.15}^{20.45}-20.93_{16.77}^{18.36}$ & $9.10_{7.03}^{11.36}-7.62_{7.27}^{24.12}$ & $2.06_{2.04}^{25.12}-13.52_{10.40}^{21.78}$ & $83.30_{2.29}^{2.84}$ \\
\hline 17 & ST Pup & $2.28_{2.29}^{32.70}-26.07_{17.27}^{15.63}$ & $9.33_{8.53}^{18.19}-36.91_{27.32}^{18.59}$ & $10.69_{6.94}^{6.40}-9.14_{8.28}^{19.19}$ & $0.43_{0.43}^{11.60}-5.15_{4.49}^{14.65}$ & $56.07_{4.14}^{4.32}$ \\
\hline 18 & SU Gem & $3.52_{3.51}^{43.03}-45.56_{32.84}^{21.97}$ & $4.68_{4.46}^{20.74}-12.39_{11.23}^{21.56}$ & $21.23_{11.60}^{01964}-5.50_{5.22}^{19.78}$ & $0.51_{0.51}^{9.92}-6.61_{5.97}^{17.57}$ & $86.85_{6.38}^{2.62}$ \\
\hline 19 & SXCen & $3.69_{3.70}^{31.56}-40.53_{23.30}^{16.94}$ & $12.07_{10.11}^{15.15}-23.07_{20.93}^{22.39}$ & $9.00_{5,24}^{7.35}-4.15_{4.08}^{16.22}$ & $0.84_{0.84}^{7.80}-6.64_{5.77}^{12.72}$ & $75.85_{3.51}^{3.100}$ \\
\hline 20 & TW Cam & $2.26_{2.27}^{44.45}-56.52_{44.46}^{26.81}$ & $0.02_{0.02}^{0.00}-4.65_{4.63}^{38.76}$ & $17.29_{11.45}^{33.38}-8.64_{8.50}^{40.91}$ & $0.82_{0.82}^{13.00}-9.79_{8.79}^{35.32}$ & $90.61_{2.14}^{2.12}$ \\
\hline 21 & UY CMa & $0.94_{0.94}^{30.95}-14.75_{11.31}^{44.58}$ & $8.63_{7.64}^{18.62}-45.36_{25.12}^{17.50}$ & $15.63_{8.53}^{9.36}-8.54_{7.81}^{16.88}$ & $0.33_{0.33}^{5.66}-5.82_{5.06}^{81.94}$ & $79.11_{1.97}^{2.10}$ \\
\hline
\end{tabular}


C. Gielen et al.: SPITZER survey of dust grain processing in stable discs around binary post-AGB stars, Online Material p 2

\section{Appendix B: Figures}
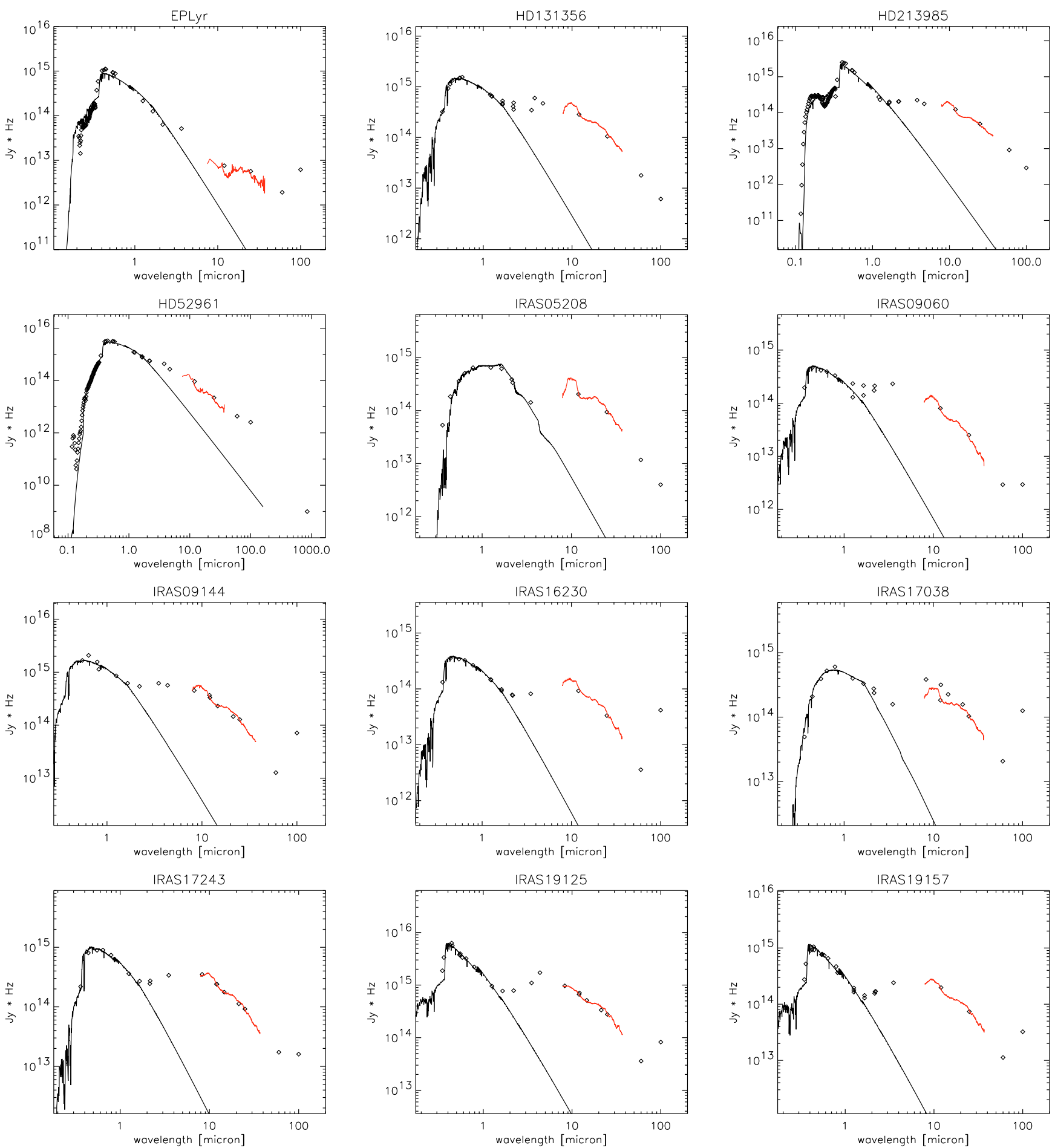

Fig. B.1. The SEDs of our sample stars. The dereddened fluxes (diamonds) are given together with the scaled photospheric Kurucz model (solid black line). The corresponding TIMMI2 and SPITZER spectrum are overplotted in red. 
C. Gielen et al.: SPITZER survey of dust grain processing in stable discs around binary post-AGB stars, Online Material p 3
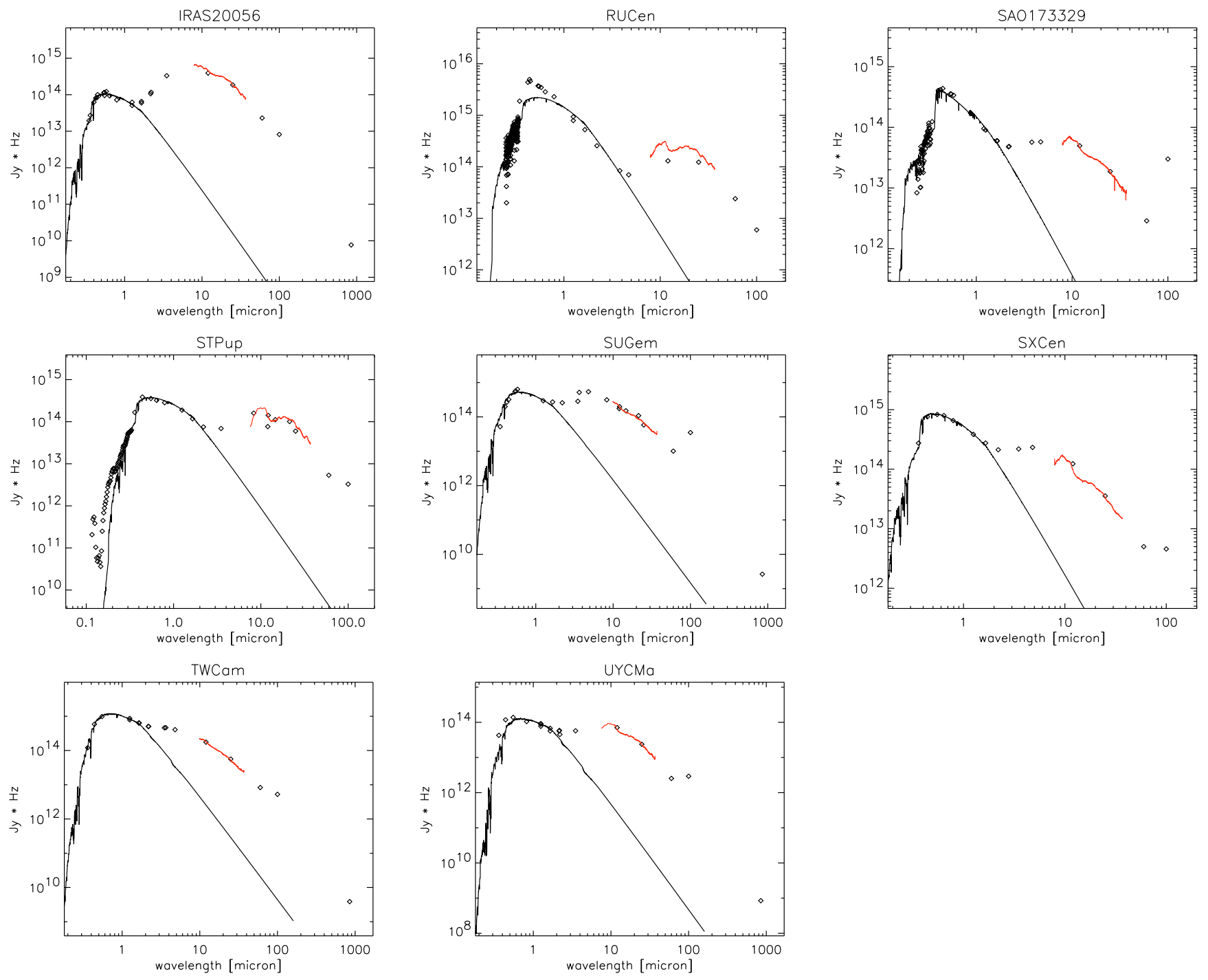

Fig. B.2. See previous caption. 


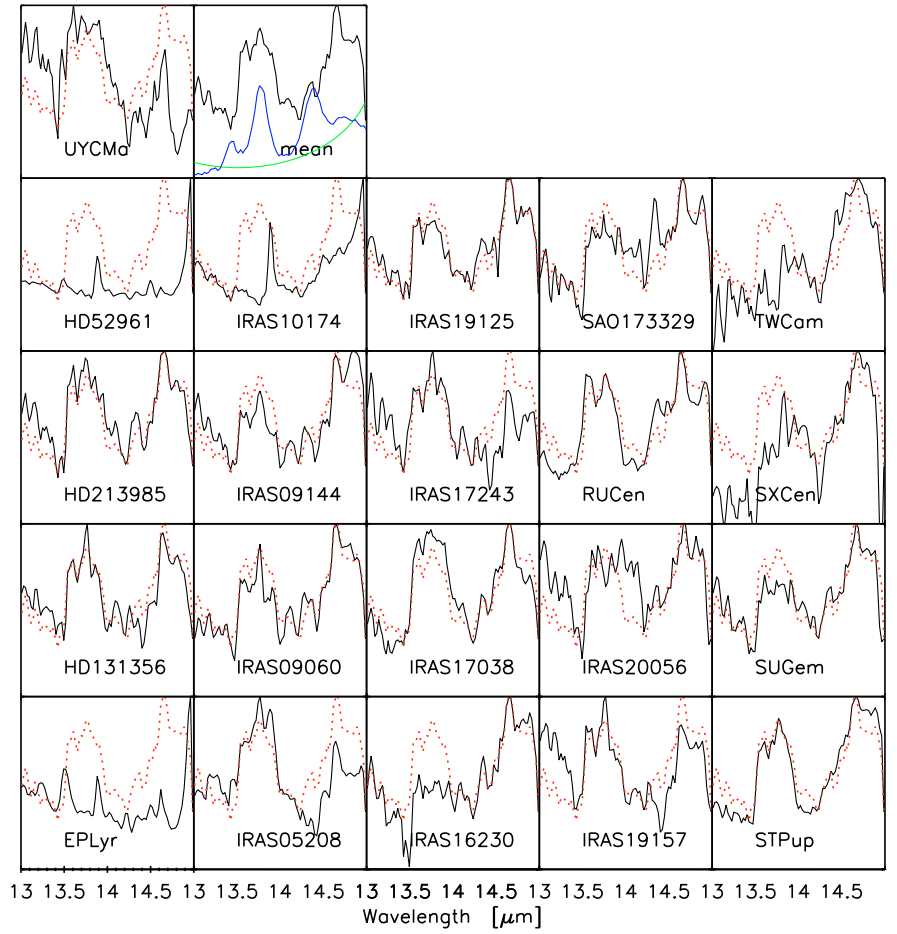

Fig. B.3. The $14 \mu \mathrm{m}$ complex, continuum subtracted and normalised. Overplotted in dotted line (red) the mean spectrum. The mass absorption coefficients of forsterite and enstatite (in CDE approximation) are plotted in green and blue.

\section{B.1. Mean spectra and complexes}

\section{B.2. The $14 \mu \mathrm{m}$ complex $(13-15 \mu \mathrm{m})$}

In Fig. B. 3 the $14 \mu \mathrm{m}$ complexes are plotted. The mean spectrum has two strong emission peaks, around 13.7 and $14.7 \mu \mathrm{m}$. The profile at $13.7 \mu \mathrm{m}$ seams to blend of two peaks, of which one can be identified as enstatite. The small feature in the mean spectrum at $14.3 \mu \mathrm{m}$ is also due to enstatite. The feature around $14.7 \mu \mathrm{m}$ remains unidentified. It seems to be made up of a broader feature from $14.3 \mu \mathrm{m}$ till $15 \mu \mathrm{m}$, with a strong narrow peak at $14.7 \mu \mathrm{m}$. In the sample stars, there is some variation in the ratio between the two strong peaks at 13.7 and $14.7 \mu \mathrm{m}$, meaning they are probably due to different dust species.

\section{B.3. The $16 \mu \mathrm{m}$ complex $(15-17 \mu \mathrm{m})$}

In Fig. B.4 the $16 \mu \mathrm{m}$ complexes are plotted. The mean spectrum shows a clear broad feature around $16 \mu \mathrm{m}$. The shape and strength is very similar to the forsterite feature at $16.2 \mu \mathrm{m}$, with a contribution of enstatite at $15.3 \mu \mathrm{m}$. On top of this broad band more narrow features at $15.9 \mu \mathrm{m}$ and $16.2 \mu \mathrm{m}$ can be discriminated. The small peaks at $15.4 \mu \mathrm{m}$ and $16.2 \mu \mathrm{m}$ are due to $\mathrm{CO}_{2}$ gas emission, which can only be clearly seen in EP Lyr, HD 52961 and IRAS 10174.

Most sample sources show a similar profile as the mean spectrum, although the ratio between the two main contributors sometimes differs, due to a different enstatite/forsterite ratio. Stars with a weak $16 \mu \mathrm{m}$ feature (like TW Cam) show a very clear separation between the three features, with widths of about $0.3 \mu \mathrm{m}$. This shows that the mean spectrum probably consist of a very broad forsterite feature topped with more narrow features of another dust (or gas?) species. HD 52961 has a strong profile

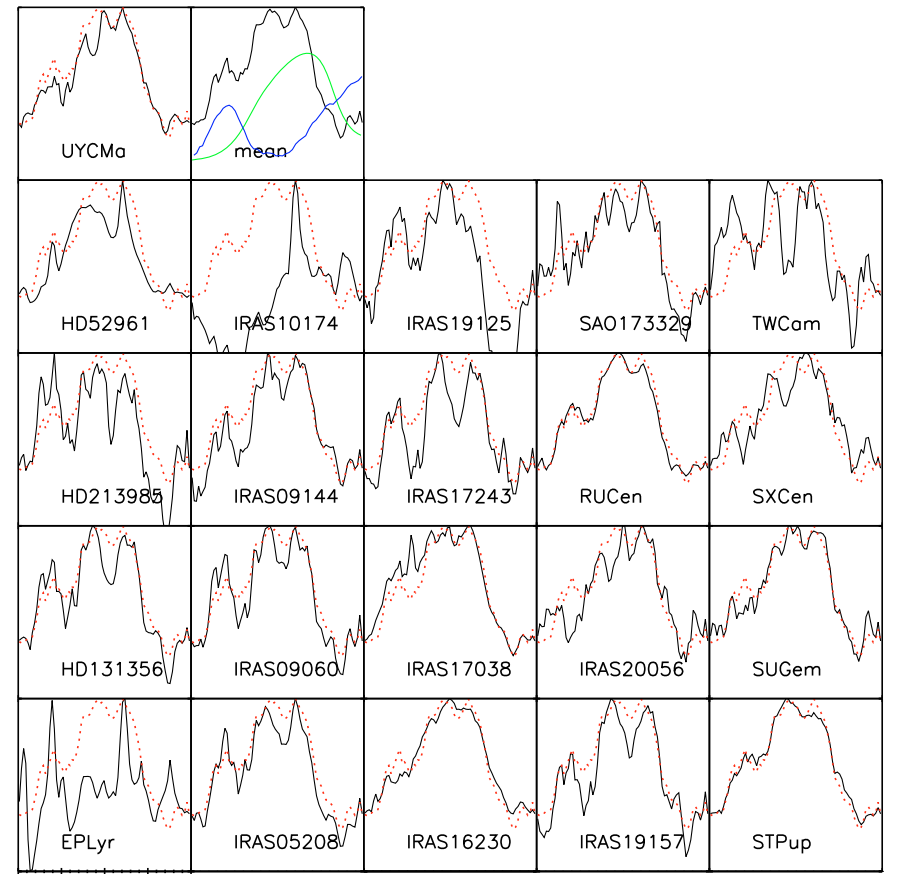

$1515.516 \quad 16.51515 .51616 .51515 .51616 .51515 .516 \quad 16.51515 .51616 .5$ Wovelength $[\mu \mathrm{m}]$

Fig. B.4. The $16 \mu \mathrm{m}$ complex, continuum subtracted and normalised. Overplotted in dotted line (red) the mean spectrum. The mass absorption coefficients of forsterite and enstatite (in CDE approximation) are plotted in green and blue.

that is shifted bluewards in comparison to the mean spectrum, with clear $\mathrm{CO}_{2}$ emission lines.

\section{B.4. The $19 \mu \mathrm{m}$ complex $(17-21 \mu \mathrm{m})$}

In Fig. B.5 the $19 \mu \mathrm{m}$ complexes are plotted. The very broad profile is again a blend of forsterite and enstatite. The sometimes very sharp feature around $19.7 \mu \mathrm{m}$ is a data reduction artefact. Most stars have the same profile shape, except for HD 52961, EP Lyr and IRAS 10174. The first two are clearly outliers in our sample stars, since they have very distinct spectra, very different from other sample stars. Looking at the Spitzer spectrum of IRAS 10174, this star has almost no crystalline features and the observed complex is actually due to poor continuum subtraction and normalisation.

\section{B.5. The $23 \mu \mathrm{m}$ complex $(20-27 \mu \mathrm{m})$}

In Fig. B.6 the $23 \mu \mathrm{m}$ complexes are plotted. The mean profile is dominated by forsterite and there is an extremely good agreement between the mean spectrum and sample stars. Remarkable little variation is detected in the whole sample.

\section{B.6. The $33 \mu \mathrm{m}$ complex $(31-37 \mu \mathrm{m})$}

In Fig. B.7 the $33 \mu \mathrm{m}$ complexes are plotted. The observed profile is mainly due to forsterite at $33.6 \mu \mathrm{m}$. At $32.5 \mu \mathrm{m}$ another feature can be observed. The ratio $32.5 / 33.6 \mu \mathrm{m}$ varies slightly from source to source, indicating that a different dust species is responsible for the feature at $32.5 \mu \mathrm{m}$. In ST Pup, the feature is clearly broader than in other sample sources. The bump at $35.7 \mu \mathrm{m}$ is not a reliable result since it sits at the end of the spectrum. 
C. Gielen et al.: SPITZER survey of dust grain processing in stable discs around binary post-AGB stars, Online Material p 5

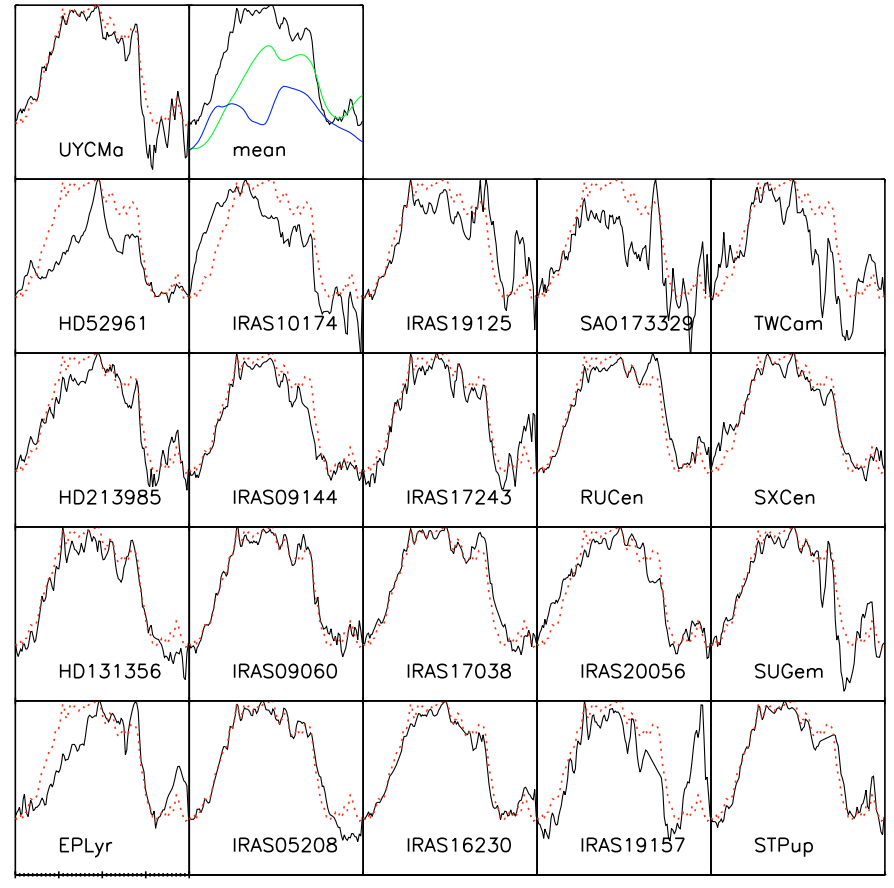

$\begin{array}{lllllllllllllllllllll}18 & 19 & 20 & 21 & 18 & 19 & 20 & 21 & 18 & 19 & 20 & 21 & 18 & 19 & 20 & 21 & 18 & 19 & 20 & 21\end{array}$

Fig. B.5. The $19 \mu \mathrm{m}$ complex, continuum subtracted and normalised. Overplotted in dotted line (red) the mean spectrum. The mass absorption coefficients of forsterite and enstatite (in CDE approximation) are plotted in green and blue.

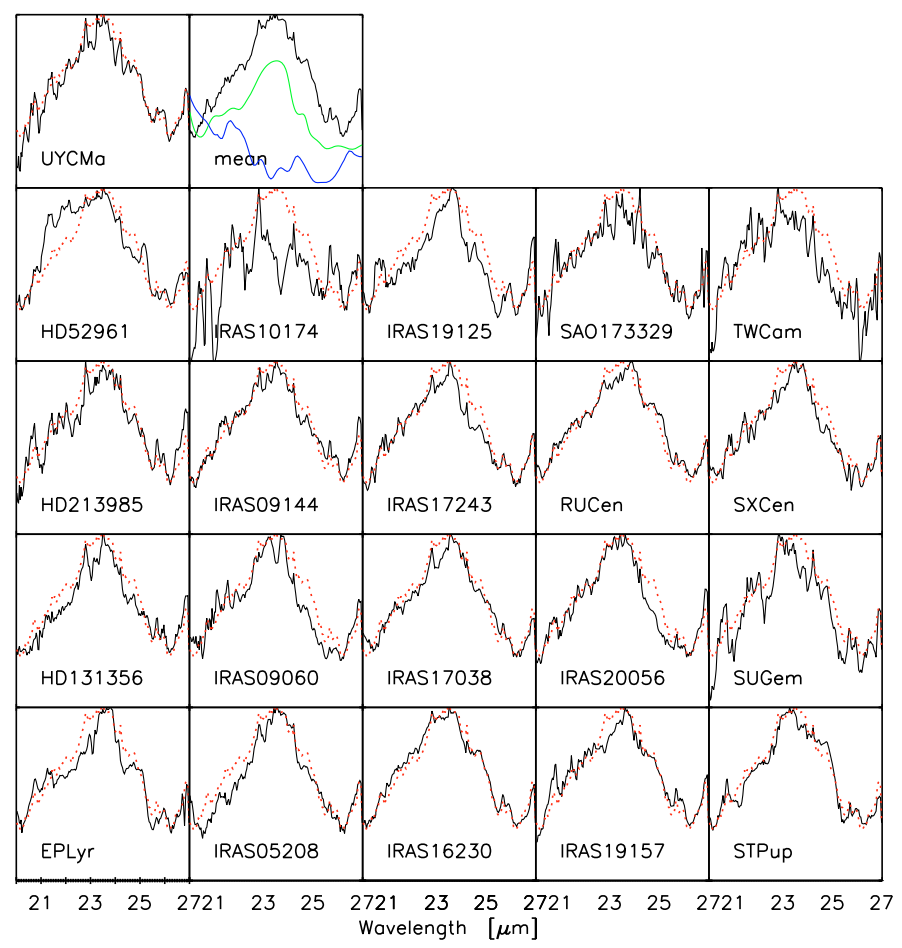

Fig. B.6. The $23 \mu \mathrm{m}$ complex, continuum subtracted and normalised. Overplotted in dotted line (red) the mean spectrum. The mass absorption coefficients of forsterite and enstatite (in CDE approximation) are plotted in green and blue.

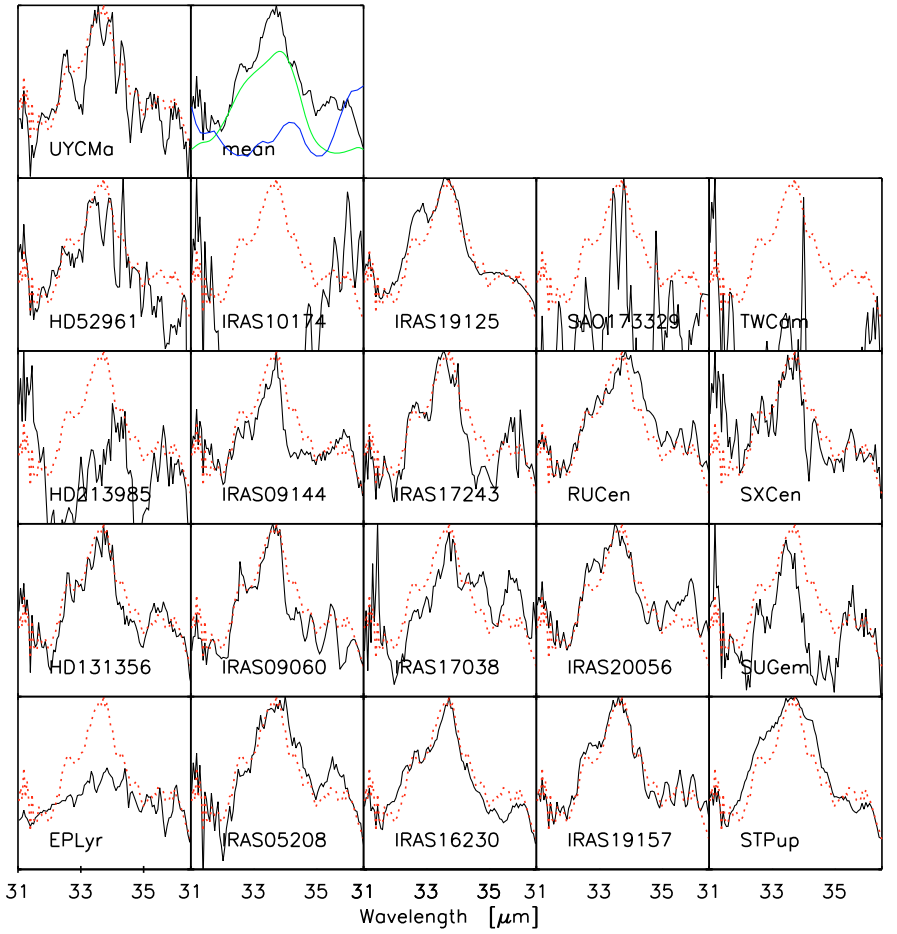

Fig. B.7. The $33 \mu \mathrm{m}$ complex, continuum subtracted and normalised. Overplotted in dotted line (red) the mean spectrum. The mass absorption coefficients of forsterite and enstatite (in CDE approximation) are plotted in green and blue. 
C. Gielen et al.: SPITZER survey of dust grain processing in stable discs around binary post-AGB stars, Online Material p 6
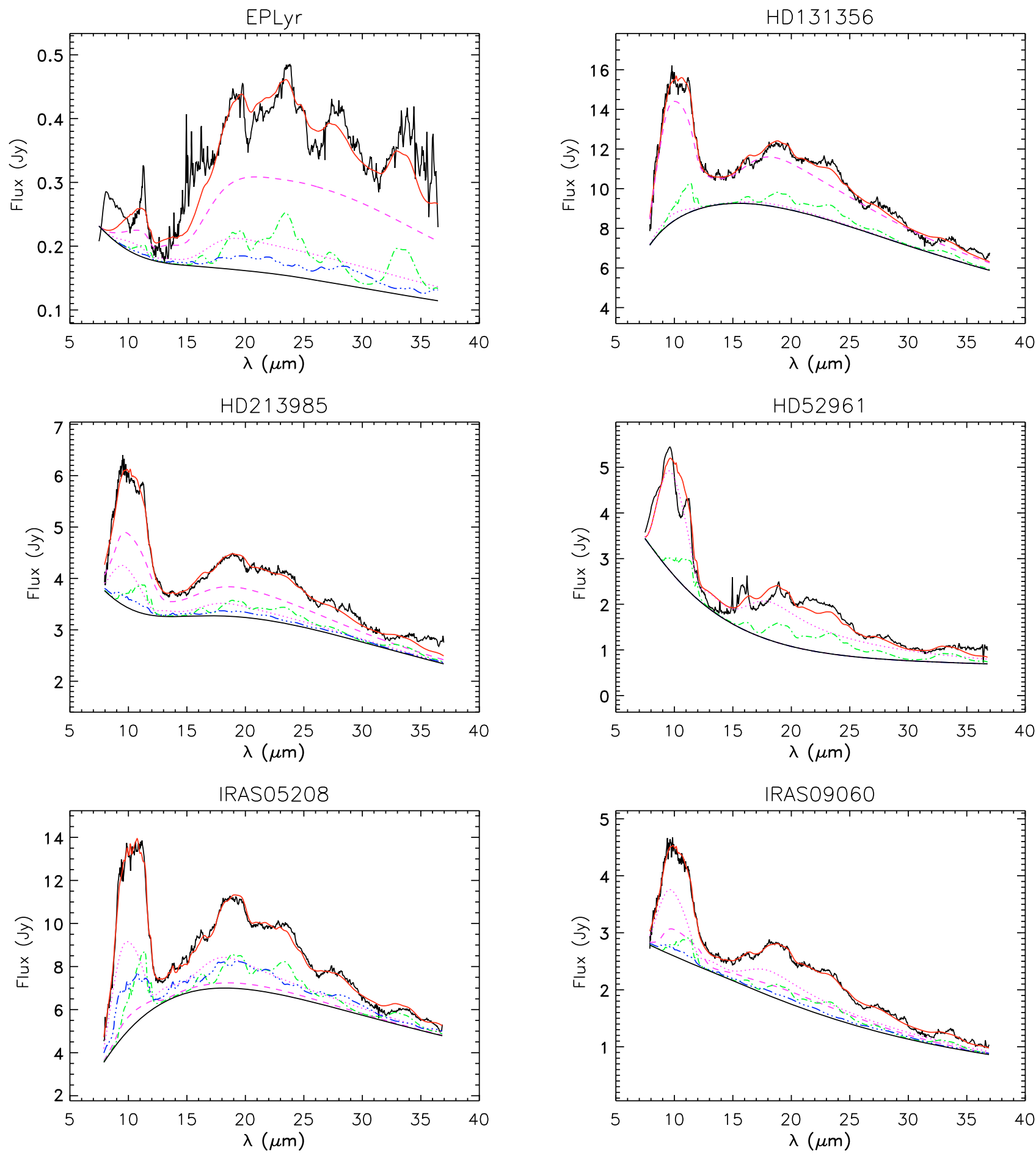

Fig. B.8. Best model fits for our sample stars, showing the contribution of the different dust species. The observed spectrum (black curve) is plotted together with the best model fit (red curve) and the continuum (black solid line). Forsterite is plotted in dash-dot lines (green) and enstatite in dash-dot-dotted lines (blue). Small amorphous grains $(2.0 \mu \mathrm{m})$ are plotted as dotted lines (magenta) and large amorphous grains $(4.0 \mu \mathrm{m})$ as dashed lines (magenta). 
C. Gielen et al.: SPITZER survey of dust grain processing in stable discs around binary post-AGB stars, Online Material p 7

IRAS09144

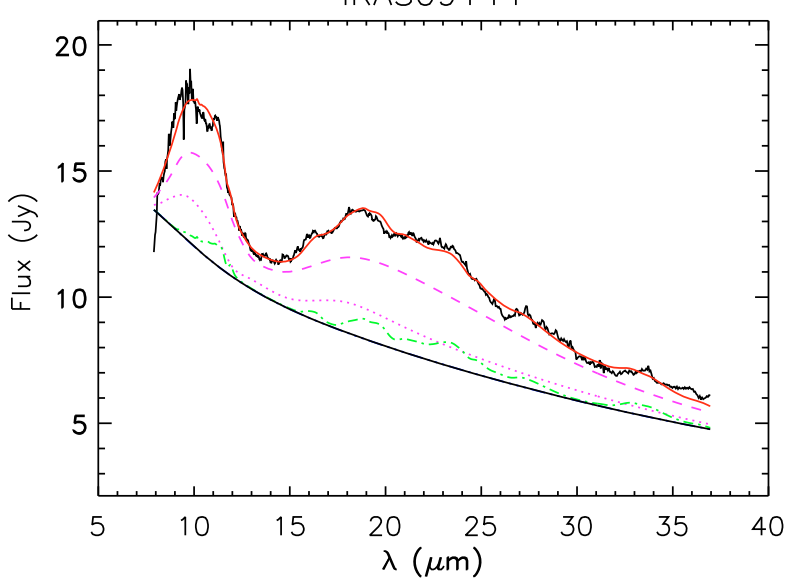

IRAS 16230

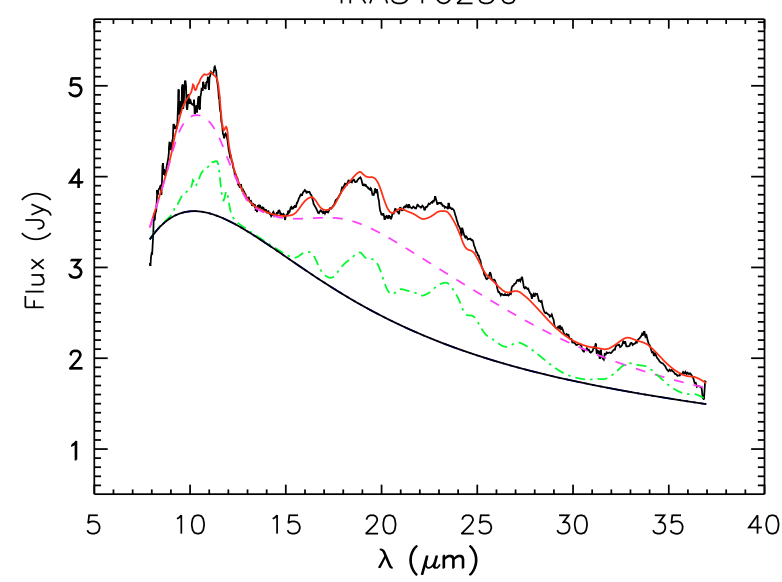

IRAS 17243

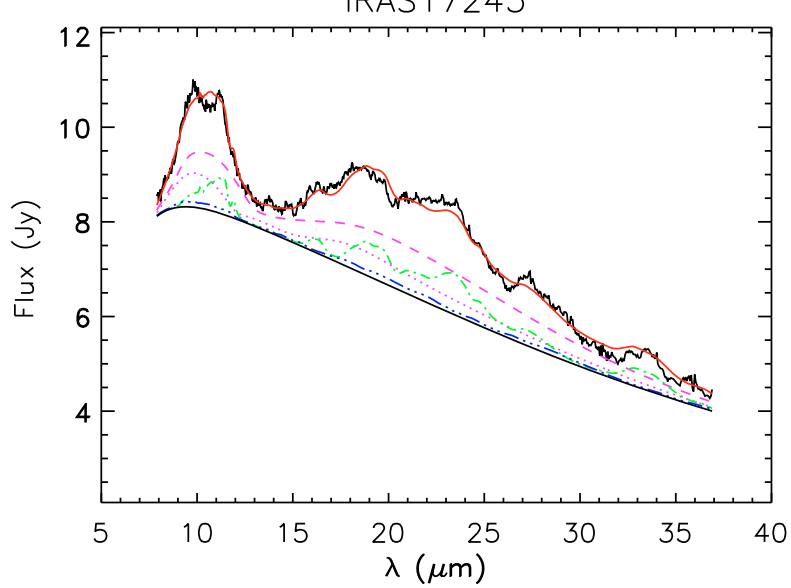

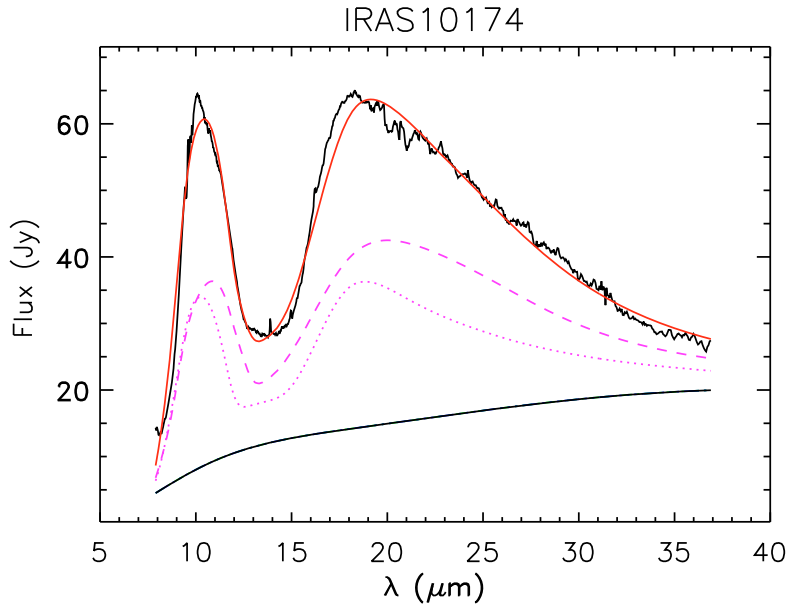

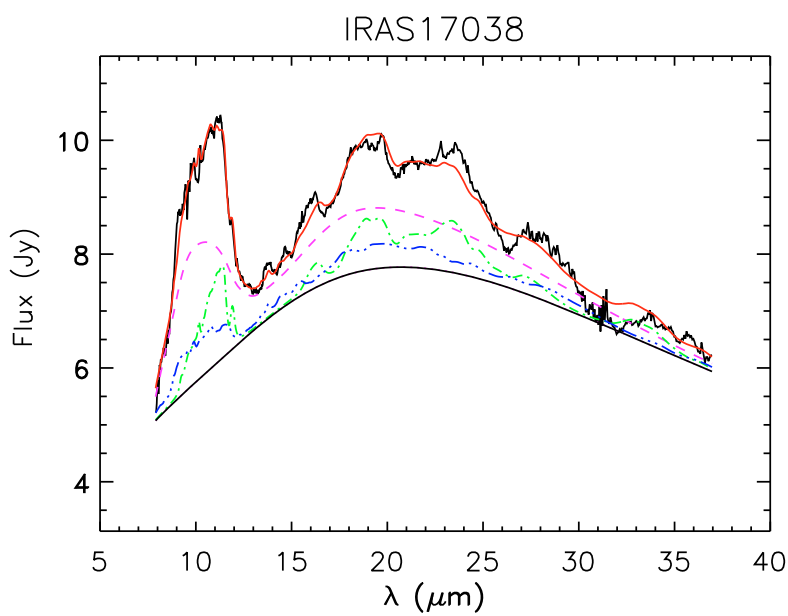

IRAS 19125

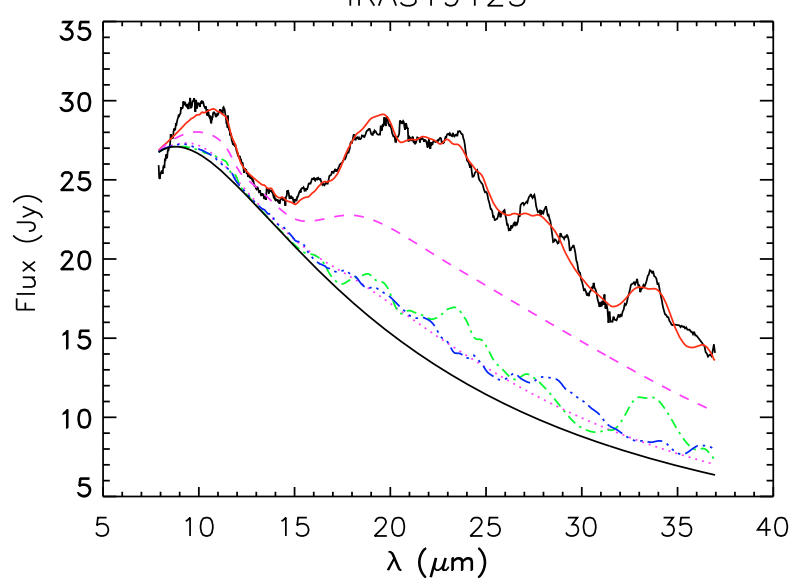

Fig. B.9. See previous caption. 
C. Gielen et al.: SPITZER survey of dust grain processing in stable discs around binary post-AGB stars, Online Material $p 8$
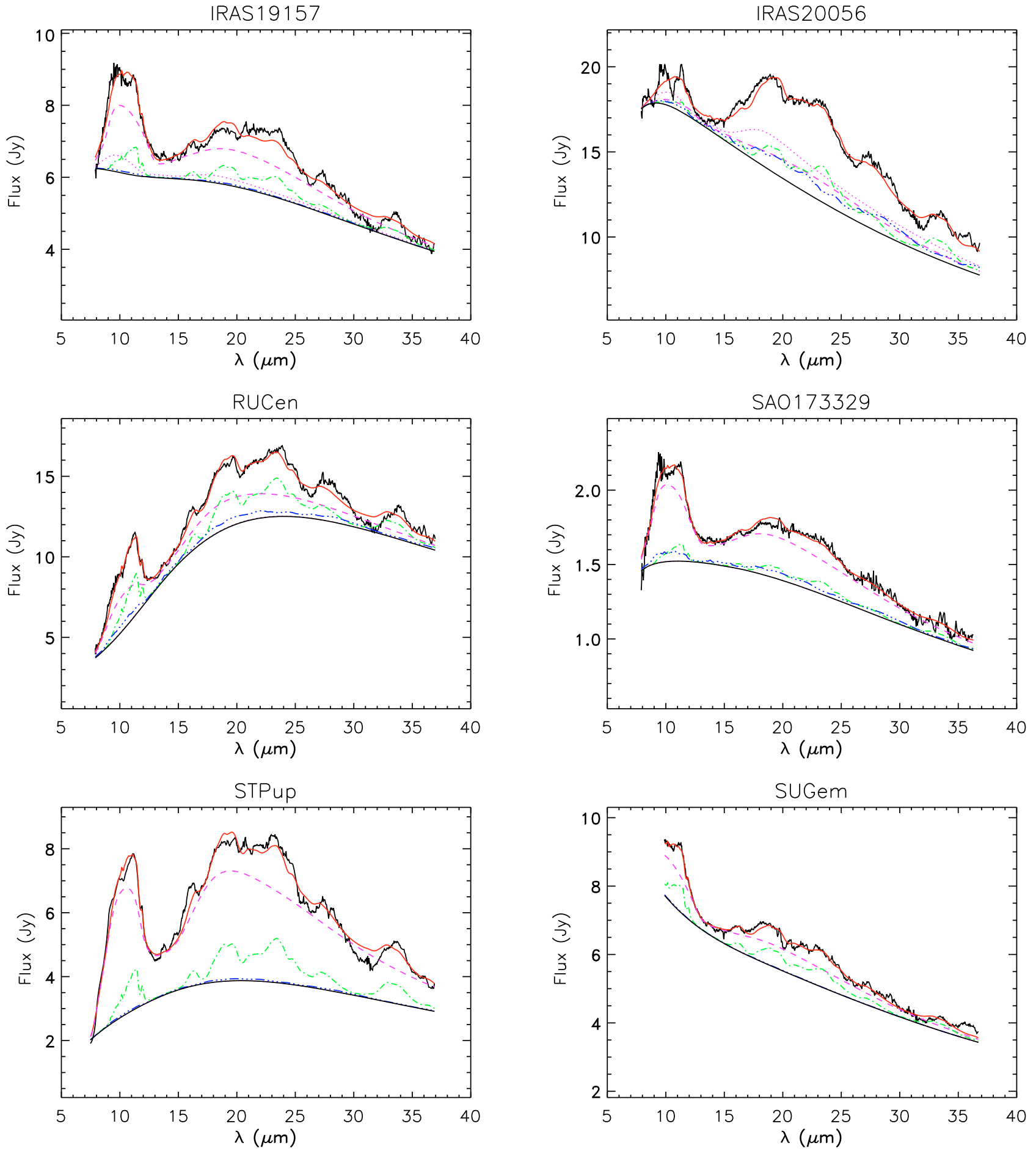

Fig. B.10. See previous caption. 
C. Gielen et al.: SPITZER survey of dust grain processing in stable discs around binary post-AGB stars, Online Material p 9
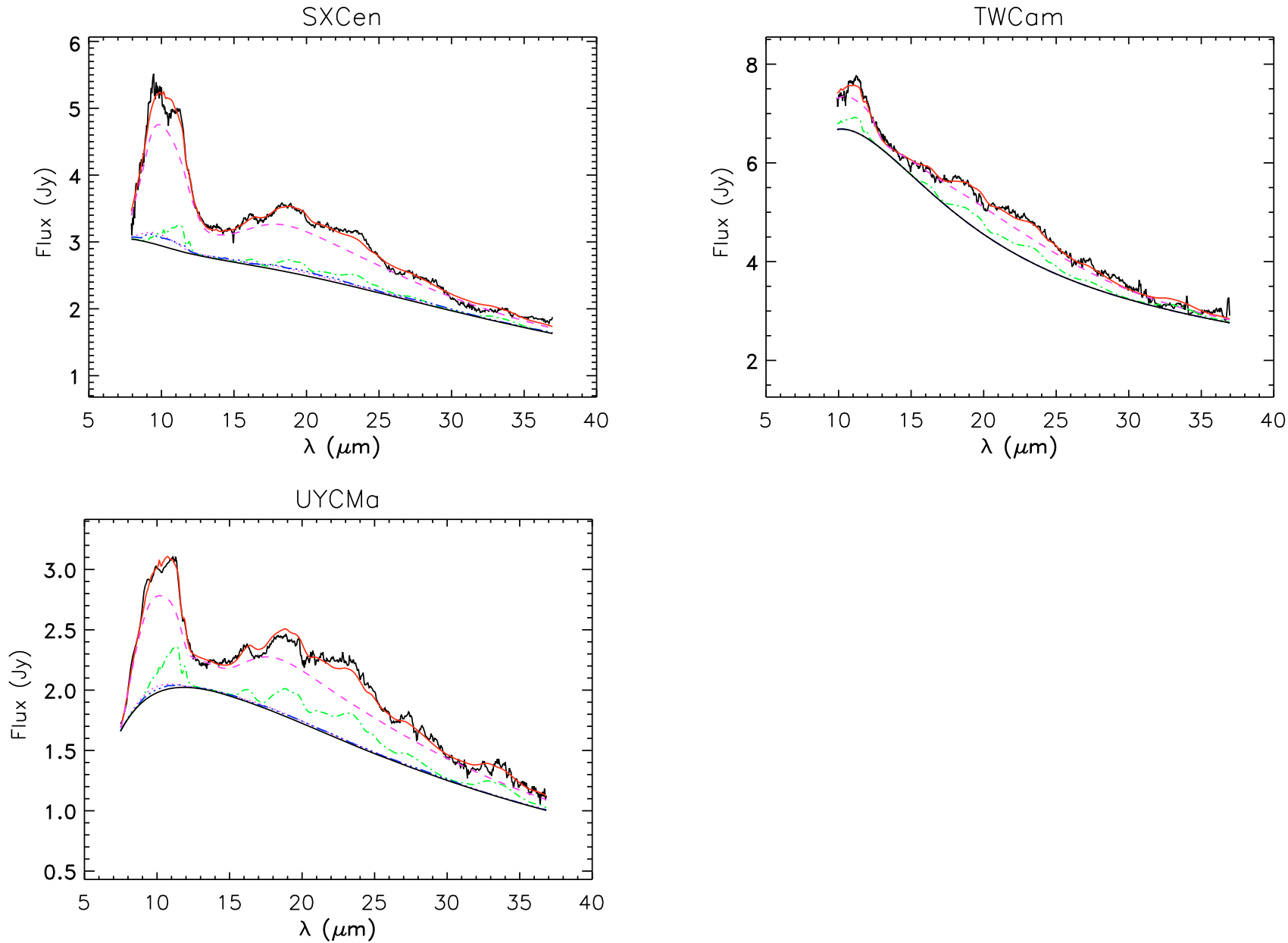

Fig. B.11. See previous caption. 